\title{
Detection of steam in the circumstellar disk around a massive Young Stellar Object ${ }^{\star}$
}

\author{
W.-F. Thi ${ }^{1,2}$ and A. Bik ${ }^{1,3}$ \\ 1 Sterrenkundig Instituut Anton Pannekoek, University of Amsterdam, Kruislaan 403, 1098 SJ Amsterdam, The Netherlands \\ e-mail: thi@science.uva.nl \\ 2 ESA Research and Scientific Support Department, ESTEC, Keplerlaan 1, 2201 AZ Noordwijk, The Netherlands \\ 3 European Southern Observatory, Karl-Schwarzschild-Strasse 2, 85748 Garching, Germany
}

Received 20 October 2004 / Accepted 18 March 2005

\begin{abstract}
We report on the observation of hot water vapor (steam) in the inner AU of a young massive star located in the starforming region IRAS 08576-4334. The water lines are detected in a medium resolution $(R \sim 10000) K$-band spectrum taken by the infrared spectrometer ISAAC mounted on the VLT-ANTU. The water vapor is at a mean temperature of $1565 \pm 510 \mathrm{~K}$, cooler than the hot $\mathrm{CO}$ found in the same object, which is at $\simeq 1660 \mathrm{~K}$; and the column density is $N\left(\mathrm{H}_{2} \mathrm{O}\right)=(2.5 \pm 0.4) \times 10^{18} \mathrm{~cm}^{-2}$. The profile of both $\mathrm{H}_{2} \mathrm{O}$ and $\mathrm{CO}$ lines is best reproduced by the emission from a Keplerian disk. To interpret the data, we also investigate the formation of molecules and especially $\mathrm{CO}$ and water vapor in the inner hot and dense part of disks around young high mass stars using a pseudo time-dependent gas-phase chemical model. Molecules are rapidly photodissociated but this destruction is compensated for by an efficient formation due to fast neutral-neutral reactions. The ability of CO molecules to self-shield significantly enhances its abundance. Water molecules are sufficiently abundant to be detectable. The observed $\mathrm{H}_{2} \mathrm{O} / \mathrm{CO}$ ratio is reproduced by gas at $1600 \mathrm{~K}$ and by an enhanced UV field over gas density ratio $I_{\mathrm{UV}} / n_{\mathrm{H}}=10^{-4}-10^{-6}$. The simulations support the presence of $\mathrm{CO}$ and $\mathrm{H}_{2} \mathrm{O}$ molecules in the inner disks around young massive stars despite the strong $\mathrm{UV}$ radiation and show that the $\mathrm{OH}$ radical plays an essential role in hot gas chemistry.
\end{abstract}

Key words. stars: formation - astrochemistry - circumstellar matter

\section{Introduction}

Disks are found around many low-mass young stars and are natural byproducts of the star formation process (e.g., Waters \& Waelkens 1998; Natta et al. 2000 for the Herbig Ae stars, and Mundy et al. 2000 for the T Tauri stars). Unfortunately, observations of disks around their higher counterparts remain inconclusive (Churchwell 2002). The presence or absence of a gaseous disk may help in understanding the detailed physics of high-mass star formation. One of the main difficulties in the formation of massive stars by direct molecular core collapse is that the radiation pressure of a luminous massive protostar will halt the accretion of matter, ultimately limiting the final mass of the star (Wolfire \& Cassinelli 1987). Two major scenarii have been proposed to overcome this shortcoming. In the first one, massive stars can form by coagulation of two lower mass objects in a dense star cluster (Bonnell \& Bate 2002). In the second scenario, a massive circumstellar disk will allow a significant amount of matter to accrete to the star (Yorke \& Sonnhalter 2002; Jijina \& Adams 1996). Evidence of the presence of a dense gaseous disk close to the star NGC 2024-IRS 2

* Based on observations collected at the European Southern Observatory at La Silla and Paranal, Chile (ESO Programmes 68.C-0652 and 69.C-0448). as presented by Lenorzer et al. (2004). The main argument is that the infrared excess observed in NGC 2024-IRS 2 can be best explained by emission from a dense gaseous disk extending up to $0.6 \mathrm{AU}$.

Knowledge of the structure and composition of the inner disk is essential for our understanding of the accretion of matter onto the star. At the high densities and temperatures $(100-2000 \mathrm{~K})$ characteristic of disks at $0.1-5 \mathrm{AU}$ around young stars, molecules are expected to be sufficiently excited to produce a rich ro-vibrational spectrum in the near- and midinfrared. $\mathrm{CO}$ overtone emission, which is most likely emitted by a disk, has been detected and analyzed by Scoville et al. (1983) toward the Orion BN object and by Chandler et al. (1995) toward NGC 2024-IRS. Disk models have been successful in modeling CO bandhead emission in low-mass, as well as high-mass, young stellar objects (Najita et al. 1996; Chandler et al. 1995; Kraus et al. 2000). Cooler CO fundamental emission, which probes the planet-forming region of disks, has been detected in a large number of low-mass premain-sequence stars (Blake \& Boogert 2004; Najita et al. 2003; Brittain et al. 2003).

Recent observations of $\mathrm{CO}$ overtone emission from young early type stars further support the disk hypothesis (Bik \& Thi 2004; Blum et al. 2005). The presence of CO molecules raises 
the question of their formation and/or survival in the harsh environment close to massive stars. Molecules other than $\mathrm{CO}$ are also expected to exist in the inner few AU of disks. Carbon, nitrogen, and oxygen are the most abundant elements after Hydrogen and Helium. At high temperatures and low pressures the dominant equilibrium species of these elements are $\mathrm{CO}, \mathrm{N}_{2}$, and $\mathrm{H}_{2} \mathrm{O}$; at intermediate temperatures, $\mathrm{CH}_{4}, \mathrm{~N}_{2}$, and $\mathrm{H}_{2} \mathrm{O}$; and at low temperatures and high pressures, $\mathrm{CH}_{4}, \mathrm{NH}_{3}$, and $\mathrm{H}_{2} \mathrm{O}$ (Lewis \& Prinn 1980). In all cases water vapor is the second molecular reservoir of oxygen after $\mathrm{CO}$ in the gas phase at a temperature between its dissociation temperature $(\sim 2500 \mathrm{~K})$ and its condensation temperature $(\sim 100-150 \mathrm{~K})$. Basically, all gas-phase oxygen locked into $\mathrm{H}_{2} \mathrm{O}$ does not participate to further reactions (e.g., Kaufman \& Neufeld 1996; Elitzur 1979). Chemical models of protoplanetary disks abound in the literature for low-mass stars (e.g., Aikawa et al. 1999; Markwick et al. 2002; Semenov et al. 2004), high-mass stars (e.g., Nguyen et al. 2002) or debris-disks (e.g., Kamp et al. 2003), although most studies were limited to the region located beyond a few AU. Nevertheless, several studies focus on the thermalchemical structure of the inner disks around young low mass stars (Glassgold et al. 2004).

Fundamental ro-vibrational and pure-rotational water lines have been observed in the envelope of high-mass young stellar objects using the Short- and Long-Wavelength Spectrometer on board the Infrared Space Observatory (e.g., Boonman et al. 2003; Wright et al. 2000; Helmich et al. 1996). They find that freeze-out of water onto grains predominates in the cold part of the envelope, while the high abundance of water in the warm part can only be explained by sublimation of water molecules.

Water emission lines have also been observed in the submillimeter domain with the Submillimeter Wave Astronomy Satellite (SWAS), but the beam was too large to allow the study of small objects like the disks around high mass young stellar objects. Surprisingly, SWAS data show that gaseous $\mathrm{H}_{2} \mathrm{O}$ and $\mathrm{O}_{2}$ are not the dominant carriers of elemental oxygen in cold molecular clouds; the oxygen remains in atomic form, providing strong constraints on chemical models (Melnick et al. 2000; Bergin et al. 2000).

Highly excited water lines, which are absent in our atmosphere, are detected from the ground in the atmosphere of M dwarfs (see Allard et al. 2001, and references therein), Sunspots (Zobov et al. 2000), and in the disk around the lowmass young star SVS 13 (Carr et al. 2004). In the latter object, $\mathrm{CO}$ bandhead emission has also been detected.

In this paper, we model the hot water emission lines detected in the disk around the massive young star 08576nr292 (IRAS 08576-4334). The young stellar object (YSO) $08576 \mathrm{nr} 292$ is one of the infrared sources detected in a survey of HII regions by Kaper et al. (2005). We also investigate the condition for formation and/or survival of molecules (mainly $\mathrm{CO}, \mathrm{H}_{2} \mathrm{O}$ ) in the gaseous disk around a massive young star. The inner disk around a young massive star is probably too hot for dust grains to survive, such that no $\mathrm{H}_{2}$ formation on the grain surface can occur. Moreover, far ultraviolet photons are no longer absorbed by dust so that photodissociation occurs at high column densities. In addition, the disk temperature and density are high, making the chemistry different compared
Table 1. Properties of $08576 \mathrm{nr} 292$. The position and observed magnitude and color are taken from Kaper et al. (2005). The extinction estimate and derived unreddened magnitude and color are taken from Bik et al. (2004).

\begin{tabular}{lc}
\hline \hline \multicolumn{2}{c}{$08576 \mathrm{nr} 292$} \\
\hline RA $(\mathrm{J} 2000)$ & $08: 59: 21.6$ \\
Dec $(\mathrm{J} 2000)$ & $-43: 45: 31.6$ \\
$K(\mathrm{Mag})$ & $9.4 \pm 0.01$ \\
$J-K(\mathrm{Mag})$ & $4.7 \pm 0.09$ \\
$A_{\mathrm{V}}$ & 12 \\
$K_{0}(\mathrm{Mag})$ & -3.1 \\
$J_{0}-K_{0}(\mathrm{Mag})$ & 0.4 \\
$d(\mathrm{kpc})$ & 0.7 \\
$M_{*}\left(M_{\odot}\right)$ & 6 \\
\hline
\end{tabular}

to other environments. For example, endothermic reactions or reactions with an activation energy, mostly the neutral-neutral reactions, and three-body reactions may dominate the chemical network over the classical ion-molecule reactions. The chemistry scheme is probably closer to that in shocks or protostellar winds than that of a cold quiescent molecular cloud.

The paper is organized as follows: we first present the observations and the fit to the $K$-band spectrum in Sect. 2. Then we explain the chemical models in Sect. 3. In Sect. 4, we present and discuss the results of the numerical runs and then finally conclude in Sect. 5.

\section{Observations}

\subsection{The high-mass young star $08576 n r 292$}

Identified by Liseau et al. (1992) as IRS 34 in their survey of IRAS sources in the Vela Molecular Cloud, IRAS 08576-4334 is a high-mass star forming-region with total bolometric luminosity $\log \left(L / L_{\odot}\right)=4.35$. The distance to the cloud has been estimated to be around $700 \mathrm{pc}$. The extended HII region IRAS 08576-4334 includes a radio continuum source known as G265.151+01.454 (Walsh et al. 1999). 08576nr292 is one of the near-IR sources detected in the $J$ - and $K$-band image of IRAS 08576-4334 by Kaper et al. (2005) and discussed in Bik et al. (2004). The properties of $08576 \mathrm{nr} 292$ are listed in Table 1 . After correction by 12 mag of extinction, as derived by Bik et al. (2004), the $J_{0}-K_{0}$ color of $08576 \mathrm{nr} 292$ is small (0.4). Because the intrinsic color of hot stars is $\sim-0.2$, the near-IR excess from $08576 \mathrm{nr} 292$ is $(J-K)=0.6$, suggesting no strong hot dust emission.

\subsection{Observation and data reduction}

The spectrum of $08576 \mathrm{nr} 292$, identified in the line of sight to IRAS 08576-4334, was observed with ISAAC at the Very Large Telescope ANTU in 2002 at a resolving power of 10000 (slit width of $\left.0.3^{\prime \prime}\right)$. The near-infrared data were reduced in a standard way using a combination of the dedicated software package Eclipse and IRAF. We made use of flatfield taken during 
the night, and arc frames were taken by the European Southern Observatory (ESO) staff during the day.

Standard stars of spectral type A observed at similar airmass were used to correct for telluric $\mathrm{OH}$ emission and absorption. The wavelength calibration was achieved by using arc lines in combination with atmospheric $\mathrm{OH}$ lines. The details of the observations and datareduction are presented in Bik et al. (2004). The chosen setting covers the spectral range from 2.280 till 2.400 micron so that the four first CO bandheads $(\Delta v=2$, where $v$ is the vibrational quantum number) can be observed. At resolving power $10000\left(\Delta v \simeq 30 \mathrm{~km} \mathrm{~s}^{-1}\right)$ the profile of the lines is not resolved and a few lines may even be blended.

\subsection{Observed water lines}

The spectrum shows four prominent $\mathrm{CO}$ bandhead emissions, which are modeled in Bik \& Thi (2004). The fit to the first $\mathrm{CO}$ bandhead is shown in the lower panel of Fig. 1. The upper panel displays the spectrum shortward of $\lambda<2.2935 \mu \mathrm{m}$ in detail. The $\mathrm{CO}$ bandhead is best fitted by gas at $1660 \mathrm{~K}$ located in a Keplerian rotating disk extending from 0.2 till 3.6 AU and viewed with an angle of $20-30^{\circ}$ with respect to the disk rotation axis. The column density of $\mathrm{CO}$ is relatively large $\left(N(\mathrm{CO}) \simeq 3.9 \times 10^{21} \mathrm{~cm}^{-3}\right)$.

Several emission features can be seen blueward of the first CO bandhead (i.e. $\lambda<2.2935 \mu \mathrm{m}$ ), which we attribute to water vapor at $T>1000 \mathrm{~K}$. The emission features were checked against atmospheric transmission spectra to ensure no artifact is present. Using spectroscopic parameters drawn from the compilations HITEMP (Rothman et al. 2003) and those of Tennyson et al. (2001), most lines (often blended) in the $2.280-2.2935 \mu \mathrm{m}$ region can be assigned to hot water emission lines. HITEMP (High-Temperature molecular spectroscopic database) contains water vapor spectroscopic parameters of lines that have greater strength than $3 \times$ $10^{-27} \mathrm{~cm}^{-1} /\left(\right.$ molecule $\left.\mathrm{cm}^{-2}\right)$ at $1000 \mathrm{~K}$, while the energy of the levels in Tennyson et al. (2001) were carefully assessed using energy levels derived from sophisticated variational methods. The HITEMP database contains 1816 water lines in total between 2.278 and $2.292 \mu \mathrm{m}$, which are sufficiently strong at $T_{\text {gas }}=1500 \mathrm{~K}$, but only 10 lines have strength greater than $10^{-23} \mathrm{~cm}^{-1} /\left(\right.$ molecule $\left.\mathrm{cm}^{-2}\right)$, which is most likely the threshold for visual detection in the observed spectrum. The detected strong water lines and their spectroscopic characteristics are summarized in Table 2. The wavelength of three of the features (lines 8, 9, and 10) matches those of hot water lines detected by Carr et al. (2004) in the inner disk around the low mass young star SVS 13. The spectrum of SVS 13 obtained by Carr et al. (2004) has a higher resolution but covers a much smaller spectral range. It should be noticed that all 1816 lines are included in the synthetic spectra described in Sect. 2.4, and the position of the lines is indicated in Figs. 2 and 4.

All strong detected lines arise from the first vibrational excited symmetric (100) and antisymmetric (001) stretch level of ortho- $\mathrm{H}_{2} \mathrm{O}$. It is known that there is a near coincidence of the frequencies of those two modes. The rotational levels are high $(12<J<18)$, as expected since only high level lines are

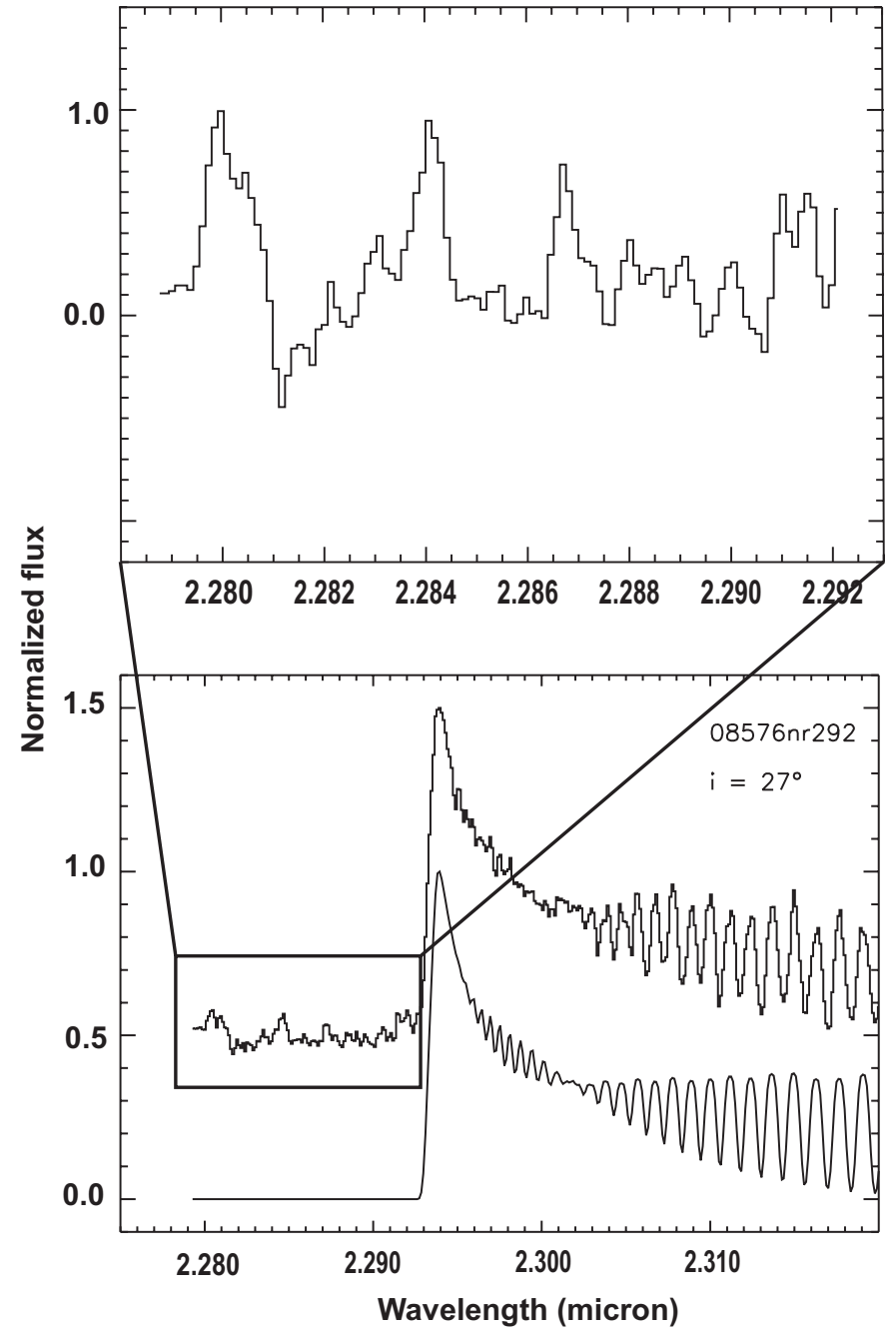

Fig. 1. The lower panel shows the best fit to the $\mathrm{CO}$ first bandhead toward $08576 \mathrm{nr} 292$. In the upper part the observed spectrum is shown. The spectrum is continuum-subtracted and normalized to the peak of the $2-0$ bandhead. The best fit of the CO-bandhead is plotted in the lower part. The parameters of the fit are discussed in Bik \& Thi (2004). Features shortward of $2.2935 \mu \mathrm{m}$ are shown in detail in the upper panel.

not present in our atmosphere and can be readily detected with ground telescopes. At the resolving power of 10000 (or resolution $\Delta v \simeq 30 \mathrm{~km} \mathrm{~s}^{-1}$ ), the lines are unresolved and few of them blended. The observed lines arise from levels with energy from $5000-9000 \mathrm{~K}$. The detected $(001)-(000)$ transitions have $\Delta K_{a}=-2$ and $\Delta K_{c}=+1$ and are therefore of $a$-type. The (100)-(000) transition is of $b$-type $\left(\Delta K_{a}=-3\right.$ and $\left.\Delta K_{c}=+1\right)$ (see Table 4).

Water lines are clearly seen in this object because they are not blended. The profile of emission lines coming from the inner part ( $<\mathrm{a}$ few $\mathrm{AU}$ ) of a disk in Keplerian rotation can have $F W H M$ up to $\simeq 200-250 \mathrm{~km} \mathrm{~s}^{-1}$ (or $\left.\Delta \lambda=0.0015 \mu \mathrm{m}\right)$ if viewed edge-on. In comparison, water lines wavelength positions are often separated by less than $65 \mathrm{~km} \mathrm{~s}^{-1}$ (or $0.0005 \mu \mathrm{m}$ ), therefore the rotational broadening of all lines produces a blend of features that can be mistaken for a continuum. 
Table 2. Strongest detected water emission lines. Various spectroscopic characteristics of the detected lines are also provided. The last column lists estimates of the critical density $n_{\text {crit }}$ assuming $T_{\text {gas }}=1000 \mathrm{~K}$ and $\sigma \simeq 10^{-15} \mathrm{~cm}^{-2}$.

\begin{tabular}{|c|c|c|c|c|c|c|c|}
\hline Line & Nuclear state & $\begin{array}{c}\operatorname{Transition}(\mathrm{u} \rightarrow \mathrm{l}) \\
\left(v_{1} v_{2} v_{3}\right) J K_{a} K_{c}-\left(v_{1}^{\prime} v_{2}^{\prime} v_{3}^{\prime}\right) J^{\prime} K_{a}^{\prime} K_{c}^{\prime}\end{array}$ & $\begin{array}{l}\text { Wavelength } \\
\text { (micron) }\end{array}$ & $\begin{array}{c}E_{\text {upper }} \\
\left(\mathrm{cm}^{-1}\right)\end{array}$ & $\begin{array}{c}E_{\text {lower }} \\
\left(\mathrm{cm}^{-1}\right)\end{array}$ & $\begin{array}{c}A_{\mathrm{ul}} \\
\left(\mathrm{s}^{-1}\right) \\
\end{array}$ & $\begin{array}{c}n_{\text {crit }} \\
\left(\mathrm{cm}^{-3}\right)\end{array}$ \\
\hline $1 \ldots \ldots$ & ortho- $\mathrm{H}_{2} \mathrm{O}$ & $(001) 15610-(000) 14411$ & 2.28020 & 7131.63 & 2746.02 & $9.50 \times 10^{-2}$ & $9.50 \times 10^{8}$ \\
\hline $2 \ldots \ldots$. & ortho- $\mathrm{H}_{2} \mathrm{O}$ & (001) 157 9-(000) 14510 & $2.28076^{(1)}$ & 7302.72 & 2918.24 & $8.88 \times 10^{-2}$ & $8.88 \times 10^{8}$ \\
\hline $3 \ldots \ldots$ & ortho- $\mathrm{H}_{2} \mathrm{O}$ & (001) $16511-(000) 15312$ & $2.28093^{(1)}$ & 7464.48 & 3080.18 & $8.27 \times 10^{-2}$ & $8.27 \times 10^{8}$ \\
\hline $4 \ldots \ldots$ & ortho- $\mathrm{H}_{2} \mathrm{O}$ & (001) $14 \quad 8 \quad 6-(000) 136 \quad 7$ & $2.28449^{(1)}$ & 7133.78 & 2756.41 & $6.56 \times 10^{-2}$ & $6.56 \times 10^{8}$ \\
\hline $5 \ldots \ldots$. & ortho- $\mathrm{H}_{2} \mathrm{O}$ & (100) $18712-(000) 17413$ (2) & $2.28462^{(1)}$ & 8394.96 & 4017.90 & $2.00 \times 10^{-1}$ & $2.00 \times 10^{9}$ \\
\hline $6 \ldots \ldots$. & ortho- $\mathrm{H}_{2} \mathrm{O}$ & (001) $1293-(000) 117 \quad 4$ & 2.28694 & 6694.58 & 2321.90 & $1.88 \times 10^{-2}$ & $1.88 \times 10^{8}$ \\
\hline $7 \ldots \ldots$. & ortho- $\mathrm{H}_{2} \mathrm{O}$ & (001) $14510-(000) 13311$ & 2.28744 & 6619.79 & 2248.06 & $6.24 \times 10^{-2}$ & $6.24 \times 10^{8}$ \\
\hline $8 \ldots \ldots$ & ortho- $\mathrm{H}_{2} \mathrm{O}$ & (001) $13 \quad 8 \quad 6-(000) 1267$ & 2.29036 & 6799.95 & 2433.80 & $4.65 \times 10^{-2}$ & $4.65 \times 10^{8}$ \\
\hline $9 \ldots \ldots$ & ortho- $\mathrm{H}_{2} \mathrm{O}$ & (001) 134 10-(000) 12211 & 2.29134 & 6139.03 & 1774.75 & $3.20 \times 10^{-2}$ & $3.20 \times 10^{8}$ \\
\hline $10 \ldots .$. & ortho- $\mathrm{H}_{2} \mathrm{O}$ & (001) $18711-(000) 17512$ & 2.29180 & 8537.65 & 4174.38 & $3.21 \times 10^{-1}$ & $3.21 \times 10^{9}$ \\
\hline
\end{tabular}

Note ${ }^{1}$ Blended lines.

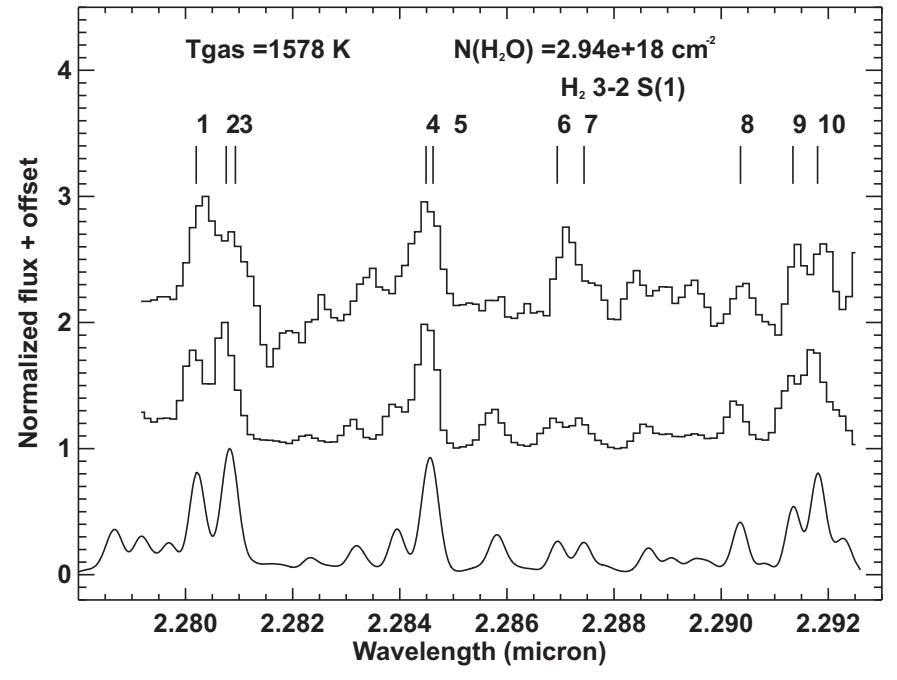

Fig. 2. Best fit to the hot water lines with the slab model. The upper spectrum is a blowup of the spectrum showed in Fig. 1 between 2.278 and $2.292 \mu \mathrm{m}$, normalized to the maximum strength at $\simeq 2.280 \mu \mathrm{m}$. The middle one represents the best fit, while the lower curve is the synthetic spectrum before degradation at the resolution of the observed spectrum. The characteristic of the strong detected lines, which are labeled 1 to 10, are given in Table 2. The $\mathrm{H}_{2} 3-2 \mathrm{~S}(1)$ emission line is located at position 6 .

\subsection{Spectral modeling}

In this section we apply two different models to fit the observed spectrum. The first model is a simplified version of the disk model applied to the $\mathrm{CO}$ bandheads of this object by Bik \& Thi (2004), and in it we assume that the gas is originating from an isothermal slab of gas. The second model used to fit the data is similar to the model used to fit the $\mathrm{CO}$ bandheads. The gas is located in a Keplerian rotating, isothermal disk. We begin describing the ro-vibrational transitions of water vapor and the transition selection rules.

\subsection{1. $\mathrm{H}_{2} \mathrm{O}$ ro-vibrational transitions}

The structure of the water molecule is described in molecular physics textbooks, e.g., Bernath (1995). As a triatomic asymmetric (near-oblate) top molecule, water has three vibrational modes and three rotational modes. The vibrational levels are characterized by three vibrational quantum numbers $v_{1}$ (symmetric stretching mode $v_{1}$ ), $v_{2}$ (bending mode $v_{2}$ ), and $v_{3}$ (asymmetric stretching mode $v_{3}$ ). The energy of the lowest vibrational levels is given in Table 3. The rotational levels are labeled using the standard asymmetric top notation $J_{K_{a} K_{c}}$, where $J$ is the main rotational quantum number, $K_{a}$ the prolate quantum number (projection of $J$ along the $a$ axis), and $K_{c}$ the oblate quantum number (projection of $J$ along the $c$ axis). Notice that $K_{a}+K_{c}=J$ or $J+1$. The rotational statistical weight is $\left(g_{J}=2 J+1\right)$. The total degeneracy is $g=g_{I} g_{J}$.

Like $\mathrm{H}_{2}, \mathrm{H}_{2} \mathrm{O}$ has two hydrogen atoms each with a nuclear spin $1 / 2$, which combine to a singlet (para- $\mathrm{H}_{2} \mathrm{O}$ ) and triplet (ortho- $\mathrm{H}_{2} \mathrm{O}$ ). Transitions between para and ortho states are highly forbidden. Ortho states have a nuclear statistical weight $g_{I}$ of 3 while para states have $g_{I}$ equal to 1 . The sum $K_{a}+K_{c}+v_{3}$ is odd for ortho states and even for para states.

The selection rules for asymmetric tops depend on the components $\left(\mu_{a}, \mu_{b}, \mu_{c}\right)$ of the permanent dipole moment vector, along the $a, b$, and $c$ principal molecular axes. The vibrational selection rules are $\Delta v=1$ for the strong fundamental transitions and $\Delta v=2,3,4, \ldots$ for the weaker overtone transitions. The rotational selection rules can be divided into three general cases given in Table 4 in addition to $\Delta J=0, \pm 1$.

\subsubsection{Slab model}

For the spectral modeling of the water lines we employ a method similar to the fit of the $\mathrm{CO}$ bandhead emission, which is best modeled by a Keplerian disk seen at low inclination with respect to the rotation axis. First we simplify the model further and assume that the water vapor lines arise from a isothermal 
Table 3. Lower energy levels of vibration of water molecule in $\mathrm{cm}^{-1}$ with ground energy level at $0 \mathrm{~cm}^{-1}$. The zero-point energy is $4500 \mathrm{~cm}^{-1}$. To convert the energy scale into $\mathrm{K}$, multiply by 1.438768 .

\begin{tabular}{llll}
\hline \hline Symmetry & $\begin{array}{c}\text { Vibrational level } \\
\left(v_{1} v_{2} v_{3}\right)\end{array}$ & $\begin{array}{c}\text { Energy } \\
\left(\mathrm{cm}^{-1}\right)\end{array}$ & Level type \\
\hline $\mathrm{A}_{1}$ & 000 & 0000.00 & ground \\
$\mathrm{A}_{1}$ & 010 & 1594.75 & fundamental \\
$\mathrm{A}_{1}$ & 020 & 3151.63 & overtone \\
$\mathrm{A}_{1}$ & 100 & 3657.05 & fundamental \\
$\mathrm{B}_{2}$ & 001 & 3755.92 & fundamental \\
$\mathrm{A}_{1}$ & 030 & 4666.79 & overtone \\
$\mathrm{A}_{1}$ & 110 & 5234.97 & combination \\
$\mathrm{B}_{2}$ & 011 & 5331.27 & combination \\
$\mathrm{A}_{1}$ & 040 & 6134.01 & overtone \\
$\mathrm{A}_{1}$ & 120 & 6775.09 & combination \\
$\mathrm{B}_{2}$ & 021 & 6871.52 & combination \\
$\mathrm{A}_{1}$ & 200 & 7201.54 & overtone \\
$\mathrm{B}_{2}$ & 101 & 7249.81 & combination \\
$\mathrm{A}_{1}$ & 002 & 7445.04 & overtone \\
\hline
\end{tabular}

Table 4. Selection rules for the ro-vibration transitions in addition to $\Delta J=0, \pm 1$. The transitions in brackets are weaker than the main transitions. For ro-vibrational transitions, the parity of $K_{a}+K_{c}+v_{3}$ has to be conserved. Transitions of type a only occur for oblate molecules, whereas transitions of type $\mathrm{c}$ are only found for prolate molecules. Both oblate and prolate molecules can have b-type transitions.

\begin{tabular}{llrr}
\hline \hline Transition & Criterion & \multicolumn{1}{c}{$\Delta K_{a}$} & \multicolumn{1}{c}{$\Delta K_{c}$} \\
\hline$a$-type & $\mu_{a} \neq 0$ & $0, \pm 2( \pm 4, \ldots)$ & $\pm 1( \pm 3, \pm 5, \ldots)$ \\
$b$-type & $\mu_{b} \neq 0$ & $\pm 1, \pm 3( \pm 5, \ldots)$ & $\pm 1, \pm 3( \pm 5, \ldots)$ \\
$c$-type & $\mu_{c} \neq 0$ & $\pm 1( \pm 3, \pm 5, \ldots)$ & $0, \pm 2( \pm 4, \ldots)$ \\
\hline
\end{tabular}

slab of gas at temperature $T_{\text {gas }}$ with column density $N\left(\mathrm{H}_{2} \mathrm{O}\right)$. The remaining free parameter is the turbulent velocity width $d v$. Values for $d v$ range from 5 to $25 \mathrm{~km} \mathrm{~s}^{-1}$. One should keep in mind that the profiles are unresolved and thus $d v$ is an illconstrained parameter. The turbulent profile is a Gaussian function. For $d v>7 \mathrm{~km} \mathrm{~s}^{-1}$, the gas is highly turbulent. The transition data are taken from the compilation HITEMP, and the level population is assumed to be at local thermodynamical equilibrium (LTE).

An order of magnitude check of this last assumption can be performed. The collisional de-excitation coefficients for the transition $\mathrm{u} \rightarrow 1$ is given by $\gamma_{\mathrm{ul}} \simeq \sigma_{\mathrm{ul}} v_{\mathrm{rel}}$, where $\sigma_{\mathrm{ul}}$ is the de-excitation cross section and $v_{\text {rel }}=\sqrt{8 k T_{\text {gas }} / \pi m_{\mathrm{H}_{2} \mathrm{O}}}=$ $10^{5} \sqrt{T_{\text {gas }} / 1000} \mathrm{~cm} \mathrm{~s}^{-1}$ is the mean relative velocity of $\mathrm{H}_{2} \mathrm{O}$ molecules. In the absence of published cross sections for the transitions considered here, we assume that $\sigma_{\mathrm{ul}}$ is close to the geometrical cross section $\sigma \sim 10^{-15} \mathrm{~cm}^{2}$ of the molecule. The rates of spontaneous emission can be estimated from the Einstein coefficients $A_{\mathrm{ul}}$, which range from $10^{-5} \mathrm{~s}^{-1}$ to $3 \times 10^{2} \mathrm{~s}^{-1}$ in the $K$-band region. The critical densities are approximately $n_{\text {crit }}=A_{\mathrm{ul}} / \gamma_{\mathrm{ul}} \simeq\left(10^{5}-3 \times 10^{12}\right) \times$ $\left(T_{\text {gas }} / 1000\right)^{-1 / 2} \mathrm{~cm}^{-3}$. The number density in the inner part of disks is higher than $10^{10} \mathrm{~cm}^{-3}$ from the fit to the $\mathrm{CO}$ bandhead in LTE model. Therefore only the strongest lines in the $K$-band are in non-LTE. More specifically, the lines detected here have moderately strong Einstein coefficients $\left(10^{-2}-10^{-1} \mathrm{~s}^{-1}\right)$ and by consequence relatively low critical densities $n_{\text {crit }}$, which are reported in the last column of Table 2 .

The best model fit to the data was obtained by minimizing the $\chi^{2}$ using a Simplex (Press et al. 1992) and a genetic algorithm method (Pikaia, Charbonneau 1995). The results of the two methods were cross-checked to ensure that no local minima were reached. The best fit using a slab model gives $\mathrm{H}_{2} \mathrm{O}$ gas at $1550 \pm 510 \mathrm{~K}$ and column density of $(2.9 \pm 0.4) \times 10^{18} \mathrm{~cm}^{-2}$. The best fit parameters are gathered in Table 5. Although the errors on the gas temperature and column density are dominated by systematics, we estimated the uncertainties in the two parameters following the method described in Bevington \& Robinson (2003). The derived errors represent here an increase of $\chi^{2}$ by 1 from its value at the minimum. Noteworthy is that the statistical significance of the errors is difficult to assess. The column density of water is 3 orders of magnitude lower than $\mathrm{CO}$, while the temperature is only slightly lower for water than $\mathrm{CO}$. The intrinsic turbulent width $d v$ is an ill-constrained parameter, although a value of $5 \mathrm{~km} \mathrm{~s}^{-1}$ has been found to provide the best results. On the other hand, the column density and gas temperature are well constrained.

\subsubsection{Disk model}

The second model includes broadening effects caused by a disk viewed with an angle of $27^{\circ}$, as found by fitting the CO bandhead. The slab model spectrum is convolved with a profile which takes the Doppler shifts caused by hot water located in a Keplerian disk into account. The disk extends from $R_{\min }$ till $R_{\max }$, which are two parameters of the model. The inclination is assumed equal to the value obtained by fitting the first CO bandhead $\left(i=27^{\circ}\right)$. Line emission from a Keplerian disk has a double-peak profile. The simple convolution procedure is justified for $\mathrm{H}_{2} \mathrm{O}$ column densities in the range $10^{18}-10^{20} \mathrm{~cm}^{-2}$ where the optical depth is $\tau \ll 1$ (see Fig. 3). The central star is assumed to have a mass of $6 M_{\odot}$ (Bik et al. 2004).

The dust sublimation radius, i.e. the radius where dust grains attain $1500 \mathrm{~K}$, should provide an upper boundary to outer radius $R_{\max }$, because beyond this radius dust opacity will dominate over the gas opacity and neither water nor CO lines can be observed. A total column density of gas of $\sim 10^{25} \mathrm{~cm}^{-2}$, derived by multiplying the $\mathrm{CO}$ column density by $10^{4}$, results in a dust opacity of $\tau \sim 500$ in the $K$-band using the extinction law of Weingartner \& Draine (2001), which assumes a standard gas-to-dust mass ratio; and $R_{V}=3.1$. A CO abundance of $10^{4}$ is reached for a large range of physical conditions (see Sect. 3). However, gas located in the upper atmosphere of the disk can be warmer than the mid-plane. The total luminosity toward $08576 \mathrm{nr} 292$ is estimated to be around $10^{3} L_{\odot}$ for a star of 
Table 5. Parameters derived from the fit by the slab and disk model. The parameters for the CO bandhead fitting are derived in Bik \& Thi (2004). Accuracy in the temperature and column density estimates is limited by systematic instead of statistical errors (see text for a fuller discussion).

\begin{tabular}{lllccccc}
\hline \hline Model & Species & $\begin{array}{c}i \\
\left({ }^{\circ}\right)\end{array}$ & $\begin{array}{c}d v \\
\left(\mathrm{~km} \mathrm{~s}^{-1}\right)\end{array}$ & $\begin{array}{c}T_{\text {gas }} \\
(\mathrm{K})\end{array}$ & $\begin{array}{c}N \\
\left(\mathrm{~cm}^{-2}\right)\end{array}$ & $\begin{array}{c}R_{\min } \\
(\mathrm{AU})\end{array}$ & $\begin{array}{c}R_{\max } \\
(\mathrm{AU})\end{array}$ \\
\hline Disk & $\mathrm{CO}$ & 27 & 5 & 1662 & $3.9 \times 10^{21}$ & 0.2 & 3.6 \\
Slab & $\mathrm{H}_{2} \mathrm{O}$ & n. a. & 25 & $1580 \pm 510$ & $(2.9 \pm 0.4) \times 10^{18}$ & n. a. & n. a. \\
Disk & $\mathrm{H}_{2} \mathrm{O}$ & 27 & 5 & $1550 \pm 510$ & $(2.1 \pm 0.4) \times 10^{18}$ & $2.0 \pm 1$ & $4.0 \pm 1$ \\
\hline
\end{tabular}

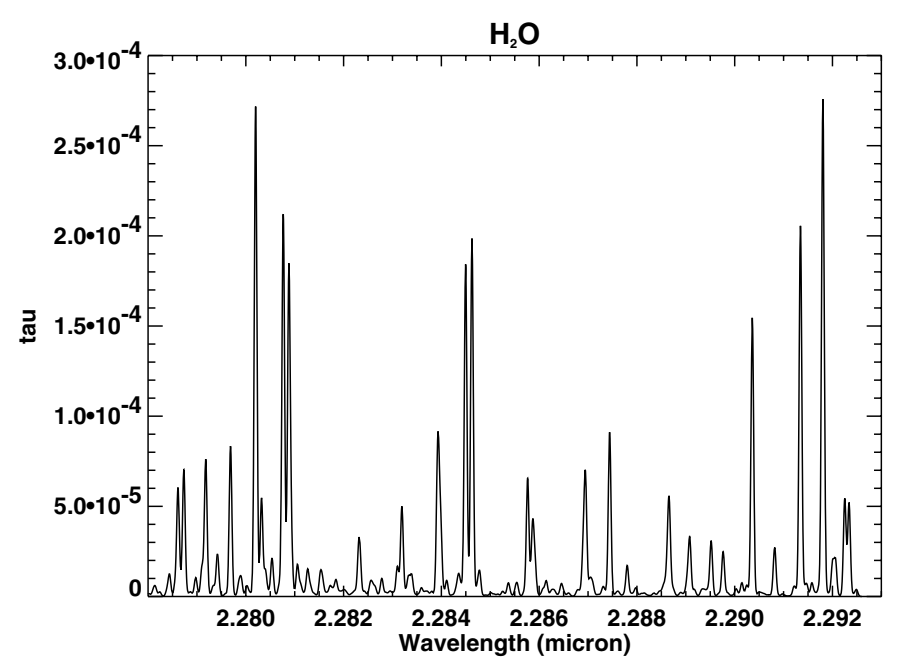

Fig. 3. Typical line optical depth for the hot water lines with $d v=$ $5 \mathrm{~km} \mathrm{~s}^{-1}, T_{\text {gas }}=1500 \mathrm{~K}$, and $N(\mathrm{CO})=3 \times 10^{18} \mathrm{~cm}^{-2}$.

mass $\simeq 6.0 M_{\odot}$. A crude estimate of the dust sublimation radius is $r_{\mathrm{d}}=0.0346 \sqrt{L_{*} / L_{\odot}} \mathrm{AU} \simeq 1 \mathrm{AU}$, where a dust sublimation temperature of $1500 \mathrm{~K}$ is assumed.

The results of the fit by a disk model is displayed in Fig. 4. The quality of the data and the resolution do not permit an accurate constraint on parameters such as $R_{\min }$ and $R_{\max }$. The gas temperature and column density are, however, better bracketed because these two parameters are set by the relative strength between lines at LTE. The best fit parameters are provided in Table 5 together with that derived from fitting the first CO bandhead.

\subsubsection{Discussion on the spectrum fitting}

The slab and disk models give similar values for the water gas temperature and column density. This closeness can be ascribed to the fact that the lines are optically thin. The intensity of optically thin emission lines is not sensitive to the actual geometry of the object. A mean value between the two models for the gas and column density is adopted in this discussion. The resulting error on the gas temperature is the scatter between the two temperatures and is much smaller than in the formal error analysis ( $15 \mathrm{~K}$ instead of $510 \mathrm{~K}$ ). The water has a mean temperature of $1565 \pm 15 \mathrm{~K}$ and column density of $(2.5 \pm 0.4) \times 10^{18} \mathrm{~cm}^{-2}$. Both models give relatively similar values for the temperature and column density. This proximity can be ascribed to the low optical thickness of the water lines.

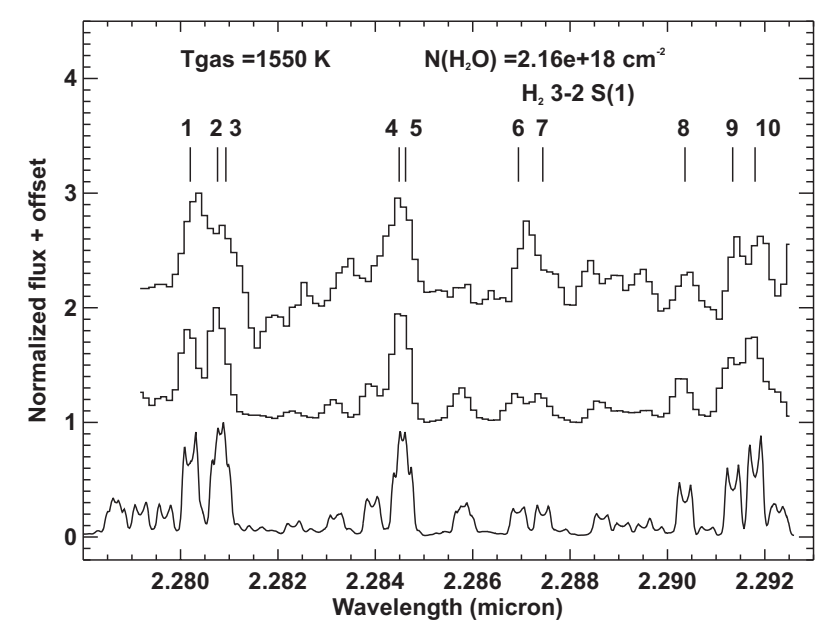

Fig. 4. Best fit to the water lines toward 08576nr292 using the disk model. The upper curve is the observed normalized and continuum subtracted spectrum. The middle one represents the best fit, while the lower curve is the synthetic spectrum before degradation at the resolution of the observed spectrum. Notice the double-peak profile for lines which are not blended.

The fits using either a slab or a disk model match the moderate signal-to-noise ratio spectrum relatively well except that both fail to reproduce the strength of the peak at $2.287 \mu \mathrm{m}$ (line 6) relative to that of at 2.2875 (line 7). The upper level energy of both lines are similar such that a non-LTE effect can be excluded (see Table 2). Moreover the Einstein $A$ coefficient is slightly lower for line 6 than 7 . Therefore line 7 should appear stronger in most situations. The most likely explanation for this discrepancy is that the observed emission at $2.287 \mu \mathrm{m}$ is a combination of hot water and $\mathrm{H}_{2} 3-2 \mathrm{~S}(1)$ emission. Indeed, a strong $\mathrm{H}_{2}$ 1-0 S(1) line has been detected in 08576nr292 at $2.218 \mu \mathrm{m}$ (Bik et al. 2004). The origin of the $\mathrm{H}_{2}$ lines is not well-determined and a large contribution from the extended HII region is probable. Alternatively, errors and uncertainties in the Einstein- $A$ coefficients may exist and can be sufficiently large to explain the bad fit around $2.287 \mu \mathrm{m}$.

The fit by a slab requires a higher turbulent width $(d v=$ $\left.25 \mathrm{~km} \mathrm{~s}^{-1}\right)$ than the disk model $\left(d v=5 \mathrm{~km} \mathrm{~s}^{-1}\right)$. This difference stems from the difficulty of constraining $d v$ with unresolved lines. The unlikely high value of $d v$ for the slab model and the simultaneous goodness of the fit to the $\mathrm{CO}$ and water lines with the Keplerian disk model favor the latter interpretation of the geometry of the hot $\mathrm{CO}$ and water emitting region. Synthetic spectra obtained with a Keplerian disk and supersonic values 
for $d v$ give unsatisfactory fits. Therefore we can rule out a turbulent Keplerian disk.

The water vapor column density of $(2.5 \pm 0.4) \times 10^{18} \mathrm{~cm}^{-2}$ and the gas temperature $T_{\text {vapor }} \simeq 1565 \mathrm{~K}$ are close to the values found around the low-mass young star SVS 13 by Carr et al. (2004), which is a priori unexpected since SVS 13 and $08576 \mathrm{nr} 292$ differ in mass. This similarity stems from that fact that the $\mathrm{H}_{2} \mathrm{O}$ emission comes from a region very close to SVS 13. Carr et al. (2004) find that the emitting area extents out to $0.3 \mathrm{AU}$, while the water vapor is emitted from 2 to $4 \mathrm{AU}$ in the disk around 08576nr292. The hot gas is located at a shorter distance from the low-mass star than from the massive star.

The water vapor temperature is about 100-200 K lower than that of the hot $\mathrm{CO}$, in accordance with the findings of Carr et al. (2004) for SVS 13, although the hot CO is much warmer around SVS 13. This lower temperature is also consistent with a large inner and outer radius (Table 5). Interestingly, the water temperature is close to the maximum dust sublimation temperature of $1500 \mathrm{~K}$, and the outer radius $R_{\min }$ is much larger than the dust sublimation radius of $1 \mathrm{AU}$, though the effect of large dust opacity in the observed spectrum is absent. In theory the low contrast between the line and continuum should have prevented any possible detection of the weak, optically thin water emission lines in the presence of dust. One possible explanation is the lack of dust grains close to the star at 1300-1500 K. The large luminosity of B5 stars can push the dust grains well beyond the dust sublimation radius through radiation pressure. Scaling the figures computed by Saija et al. (2003) to the radius, mass, and effective temperature of a B5 star, we obtain values for the ratios of radiation pressure force over gravity $\beta$ which are much larger than 10 for all types of grain compositions. We have not taken the viscous drag generated by the friction between the dust grains and the ambient gas into account, which may be important in the dense inner disk. The absence of hot dust is also compatible with the relatively small near-infrared excess $E(J-K)$. As a result of the separation between the gas and dust, the hot gas at $T \simeq 1500 \mathrm{~K}$ would be deprived of elemental oxygen that comes from the sublimation of silicate grains. Lower oxygen abundance in the gas phase may significantly affect the chemistry, but we postpone discussion of the chemistry to Sect. 4.5. Another possible explanation of the low continuum optical depth resides in the difference in dust grain lifetime. The maximum grain lifetime at a given temperature is proportional to its radius. Lamy (1974) estimated that the maximum lifetime of a $1 \mu \mathrm{m}$ radius silicate grain at $1300 \mathrm{~K}$ is $\simeq 0.6$ year and ten times shorter for a $0.1 \mu \mathrm{m}$ radius grain. Therefore, we can envision that only the larger grains in the dust size distribution can survive in the region where $T=1300-1500 \mathrm{~K}$, which results in lower dust opacity. Alternatively, if the thermal coupling between the gas and the dust is inefficient, especially in the upper atmosphere of disks, the gas can be hotter than the dust (Glassgold et al. 2004). In addition, the disk's upper atmosphere can be relatively dustfree due to dust settling. This latter possibility may be at work because a disk model with subsonic turbulent velocity is favored. Finally, it should be kept in mind that a combination of dust settling and size segregation is possible.
We have demonstrated the presence of hot $\mathrm{CO}$ and $\mathrm{H}_{2} \mathrm{O}$ in the inner $4 \mathrm{AU}$ of a Keplerian disk orbiting a B5 star. In the next section, a detailed chemical model is built in order to establish whether molecules can form and survive in the harsh environment around a hot star.

\section{Chemical modeling}

\subsection{Pure gaseous disk}

The presence of relatively large amounts of $\mathrm{H}_{2} \mathrm{O}$ and $\mathrm{CO}$ in the inner disk around young early type stars raises the question of their origin.

Early B stars are the main contributors to the mean interstellar radiation field. A critical part of the UV spectrum is the 912-1000 A range used for the computation of $\mathrm{H}_{2}$ and CO photodissociation rates. We modeled the chemistry that occurs in the HI regions using a pseudo-time dependent chemical code, i.e. all photons below $912 \AA$ have been absorbed. The main parameters of the models are the gas temperature $T_{\text {gas }}$ and density $n_{\mathrm{H}}$, the strength of the UV field and abundances of the species at time $t=0$. The temperature $\left(T_{\text {gas }}=1500-4000 \mathrm{~K}\right)$ and density range $\left(n_{\mathrm{H}}=10^{10}-10^{14} \mathrm{~cm}^{-3}\right)$ considered in our modeling correspond to the values derived by Lenorzer et al. (2004) when fitting the spectral energy distribution of NGC 2024/IRS 2.

The radiation field experienced by a parcel of gas is dominated by emission from the central star diluted by the distance to the star and by self-shielding. Because of proximity to the central star, the disk can be considered flat and the radiation flux received by the disk surface at distance $R$ from the star of radius $R_{*}$ with effective temperature $T_{*}$ is:

$F_{\mathrm{star}}=\frac{1}{2} \sigma T_{*}^{4}\left(\frac{R_{*}}{R}\right)^{2} \mu_{0}=\sigma T_{*}^{4} W_{\mathrm{disk}}$

with

$\mu_{0}=\frac{2}{3 \pi}\left(\frac{R_{*}}{R}\right)$

$\mu_{0}$ is the cosine of the angle between the incoming stellar radiation and the normal to the surface of the disk. For a typical B5V star, $R_{*}=3.9 R_{\odot}$, therefore the dilution factor $W_{\text {disk }}$ at $2 \mathrm{AU}$ is $W_{\text {disk }} \sim 7.5 \times 10^{-7}$ compared to $W_{\text {ISM }}=10^{-14}$ for the mean interstellar UV field.

The photodissociation rates in the UMIST database are multiplied by a factor $I_{\mathrm{UV}}=w_{\mathrm{disk}} / w_{\mathrm{ISM}}=7.5 \times 10^{7}$. The spectrum of the mean interstellar UV field is close to that of a B0V star, and it is assumed here that a unique enhancement factor suffices to simulate the UV photodissociation. We explore the effect of an enhanced UV field by factor $I_{\mathrm{UV}}=$ $10^{6}-10^{9}$ because of the uncertainty in the actual spectral type of $08576 \mathrm{nr} 292$ (Bik et al. 2004) and in the inner and outer radius of the emitting area. In photodominated chemistry, an important parameter is the ratio $I_{\mathrm{UV}} / n_{\mathrm{H}}=10^{-7}-10^{-2}$, which determines the behavior of the chemistry. The energy balance of high-density photodissociation regions is discussed by Burton et al. (1990). The main cooling agents of gas at a few thousand Kelvin are $\mathrm{H}_{2}$ ro-vibrational lines, followed by $\mathrm{H}_{2} \mathrm{O}$ lines. 
The heating is primarily provided by $\mathrm{H}_{2}$ vibrational heating. Far UV photons will pump $\mathrm{H}_{2}$ molecules to a bound excited electronic state (Lyman and Werner bands), from which they will fluoresce down to a high vibrational level of the ground electronic state. Most of the time $(85 \%-90 \%)$ the excited $\mathrm{H}_{2}$ molecule will fluoresce back to a low vibrational level while $10-15 \%$ of the time the molecule will dissociate. At the high densities considered in this study, the collision of excited $\mathrm{H}_{2}$ with other species contributes to the heating of the gas and thermalization of the ro-vibrational levels.

\subsection{Chemical model}

We select molecular species composed of five atoms or less and containing the elements $\mathrm{H}, \mathrm{C}, \mathrm{O}, \mathrm{N}, \mathrm{Si}, \mathrm{Mg}$, and Fe. The chemical reactions are drawn from the compilation of UMIST rate99 (Le Teuff et al. 2000). In contrast to the interstellar chemistry in cold environments, we expect that reactions with activation barrier, endothermic, and three-body reactions dominate the chemistry in inner hot disks. Therefore, besides the ion-molecules reactions, two new types of reactions are taken into account: termolecular reactions, which are catalyzed bimolecular reactions (i.e., 3-body reactions) and collider reactions, which are collision-induced dissociations. To emphasize the likely importance of 3-body reactions, we provide the dissociation sequence of water by a third body below, which is believed to be the initiation reaction in the so-called Bradford sequence:

$$
\begin{aligned}
& \mathrm{H}_{2} \mathrm{O}+\mathrm{M} \rightarrow \mathrm{OH}+\mathrm{H} \\
& \mathrm{OH}+\mathrm{CO} \leftrightarrow \mathrm{CO}_{2}+\mathrm{H} \\
& \mathrm{H}+\mathrm{H}_{2} \mathrm{O} \leftrightarrow \mathrm{OH}+\mathrm{H}_{2} \\
& \mathrm{H}+\mathrm{OH}+\mathrm{M} \rightarrow \mathrm{H}_{2} \mathrm{O}+\mathrm{M}
\end{aligned}
$$

where $\mathrm{M}$ designates a third body $\left(\mathrm{H}, \mathrm{He}, \mathrm{H}_{2}, \ldots\right)$. All together, the chemical network consists of 3299 reactions involving 301 species. Most species have negligible abundance $\left(<10^{-30}\right.$ w.r.t $\left.\mathrm{H}\right)$. No deuterated species are included in our study.

Another major difference with previous studies of chemistry in disks around low- and intermediate-mass young stars (Aikawa et al. 1999; Markwick et al. 2002; Nguyen et al. 2002), which focus on the chemistry from a few AU $(R>10 \mathrm{AU})$, is that the region modeled here is dust-free. The first consequence is the absence of the $\mathrm{H}_{2}$ formation reaction on grain surfaces. The second is that far ultraviolet photons are not absorbed by dust so that photodissociation is very effective.

\subsection{Numerical model}

The model is static and isothermal, and it numerically solves a system of differential equations that simulate the formation and destruction of atomic and molecular species. A few solvers for systems of stiff first order differential equations were used and their relative speed was checked. The solvers are GEAR, VODE (Brown et al. 1989), and two alternative algorithms
Table 6. Initial abundances. The total oxygen budget is allowed to vary to explore the effect of different $\mathrm{C} / \mathrm{O}$ elemental ratios. Water is assumed to be absent at $t=0$.

\begin{tabular}{ll}
\hline \hline Species & $n($ species $) / n(\mathrm{H})$ \\
\hline $\mathrm{H}$ & 1.0 \\
$\mathrm{He}$ & $7.1 \times 10^{-2}$ \\
$\mathrm{C}$ & $1.0 \times 10^{-4}$ \\
$\mathrm{~N}$ & $2.0 \times 10^{-5}$ \\
$\mathrm{O}$ & $1.7 \times 10^{-4}$ \\
$\mathrm{C}^{+}$ & $4.0 \times 10^{-6}$ \\
$\mathrm{CO}$ & $8.5 \times 10^{-5}$ \\
$\mathrm{H}_{2} \mathrm{O}$ & $1.0 \times 10^{-30}$ \\
\hline
\end{tabular}

described by Press et al. (1992). All solvers give similar answers for a few test models, ensuring that the results presented here are not numerical noise caused by a specific solve. The adopted solver for the results presented in this paper is VODE, the latest incarnation of the backward-differentiation method popularized by GEAR. This solver gives the fastest results on most of the models. We cover a parameter space in temperature $(1500-5000 \mathrm{~K})$, density $\left(n_{\mathrm{H}}=10^{10}-10^{14} \mathrm{~cm}^{-3}\right)$, UV field, and $\mathrm{CO}$ self-shielding factor. The initial abundance for the models is given in Table 6, and the cosmic-ray flux is set to the interstellar value. Models were run up to one year, which is more than enough for the steady-state to be reached (see next section).

\section{Results of the chemical model calculations and discussion}

After presenting the results of the chemical model calculations, we describe the formation routes of the main molecular species $\left(\mathrm{H}_{2}, \mathrm{CO}\right.$, and water) in a hot gas immersed in a strong UV field. The possibility that the molecules are formed in the outer part of the disk and transported into the inner few $\mathrm{AU}$ is discussed. Then we consider the effect of $\mathrm{CO}$ self-shielding against UV photodissociation and non-standard $\mathrm{C} / \mathrm{O}$ abundance ratio on the molecular abundances. Finally the model predictions are compared to the observed abundances.

\subsection{Results}

The abundance of $\mathrm{H}, \mathrm{H}_{2}, \mathrm{O}, \mathrm{OH}, \mathrm{H}_{2} \mathrm{O}, \mathrm{C}, \mathrm{C}^{+}, \mathrm{CO}$, and $\mathrm{N}$ are shown in Fig. 5 for a gas temperature of $1600 \mathrm{~K}$, gas density of $10^{12} \mathrm{~cm}^{-3}$, and four values of $I_{\mathrm{UV}}\left(10^{6}, 10^{7}, 10^{8}\right.$, $10^{9}$ ), neither $\mathrm{CO}$ nor $\mathrm{H}_{2}$ self-shielding. First of all, it is clear that steady-state is reached in less than a year. This rapidity is reassuring because the matter located in the inner few $\mathrm{AU}$ of disks rapidly falls onto the central star. As a comparison, the rotational period of the gas at $1 \mathrm{AU}$ around a $6 M_{\odot}$ star is $\simeq 150$ days. In most cases, steady-state is attained in a few days. The dominant species are the atomic $\mathrm{H}, \mathrm{O}, \mathrm{C}$, $\mathrm{C}^{+}, \mathrm{N}$. Molecular hydrogen $\mathrm{H}_{2}, \mathrm{CO}, \mathrm{OH}$, and $\mathrm{H}_{2} \mathrm{O}$ are the most abundant radicals/molecules. Important abundance ratios 

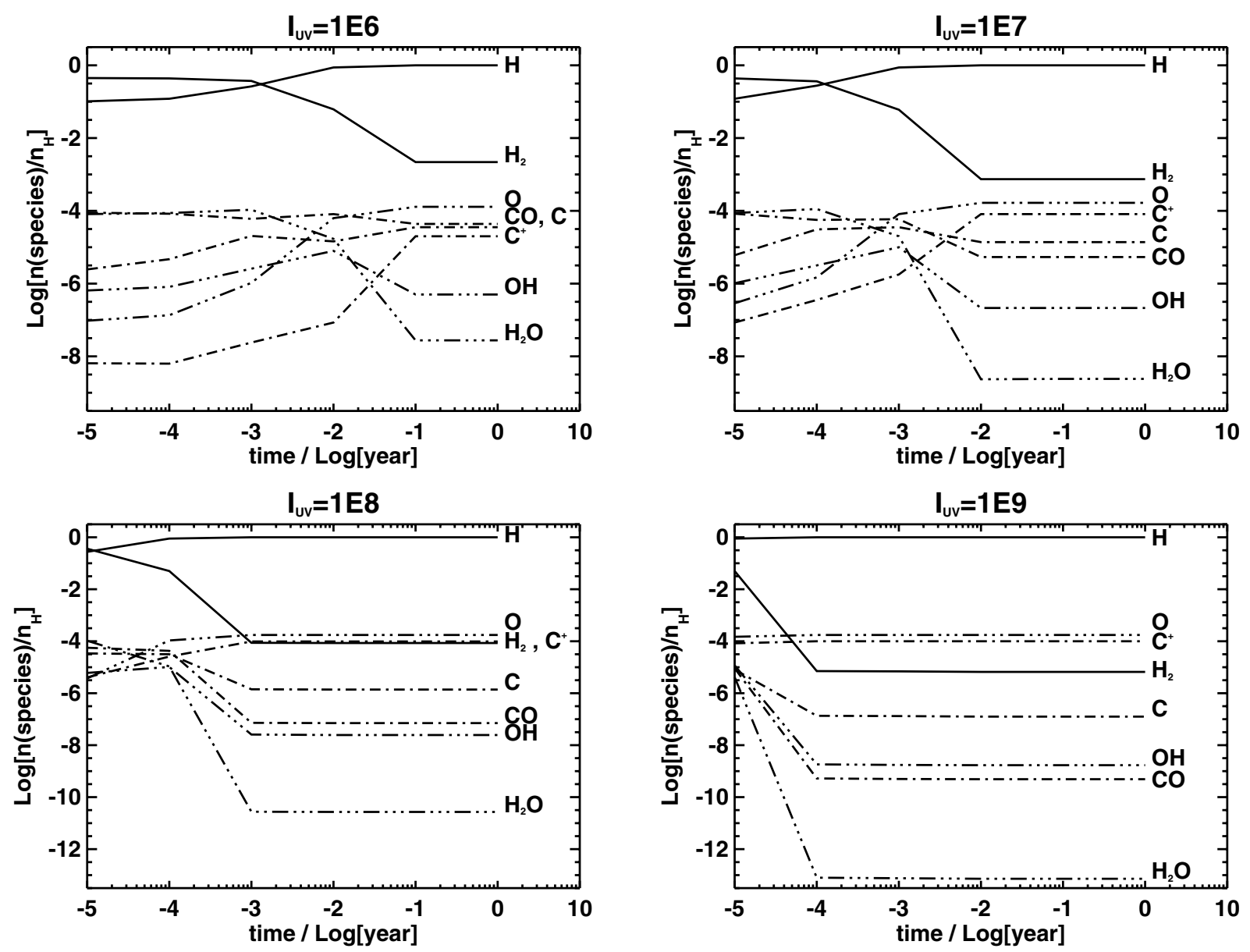

Fig. 5. Relative abundance of various species as a function of time and enhanced interstellar UV field $I_{\mathrm{UV}}$. The gas is free of dust grains has a temperature of $1600 \mathrm{~K}$ and has a density of $10^{12} \mathrm{~cm}^{-3}$ as deduced from the fitting to the $\mathrm{CO}$ and $\mathrm{H}_{2} \mathrm{O}$ emission lines. CO self-shielding was not included in these models.

such as $n(\mathrm{H}) / n\left(\mathrm{H}_{2}\right), n(\mathrm{OH}) / n\left(\mathrm{H}_{2} \mathrm{O}\right)$, and $n(\mathrm{CO}) / n\left(\mathrm{H}_{2} \mathrm{O}\right)$ are displayed in Fig. 7 for different combinations of $I_{\mathrm{UV}} / n_{\mathrm{H}}$ and $T_{\text {gas }}$ $(1600,2000,3000$ and $4000 \mathrm{~K})$. At $I_{\mathrm{UV}} / n_{\mathrm{H}}<10^{-5}$, the chemistry is photo-regulated similar to a photodissociation region, and all newly formed molecules are instantaneously destroyed. In the thermal region $\left(I_{\mathrm{UV}} / n_{\mathrm{H}}>10^{-5}\right)$, the abundance of $\mathrm{CO}$, $\mathrm{OH}$, and $\mathrm{H}_{2} \mathrm{O}$ reduces with increasing gas temperature because, once formed, those molecules react further to synthesize other species. Therefore, the optimal temperature for molecular abundance is around $2000 \pm 500 \mathrm{~K}$.

\subsection{Hot dense gas chemical network}

The chemical code monitors the main formation and destruction reactions at designated times. The formation routes for the main molecules are discussed below and a schematic is shown in Fig. 6. The chemical routes are different for dense and extremely dense gases. Below densities of around $10^{9} \mathrm{~cm}^{-3}$ and at high temperature, the chemistry is dominated by two-body reactions with activation energy of the order of few thousand Kelvin, whereas at very high densities, three-body reactions dominate.

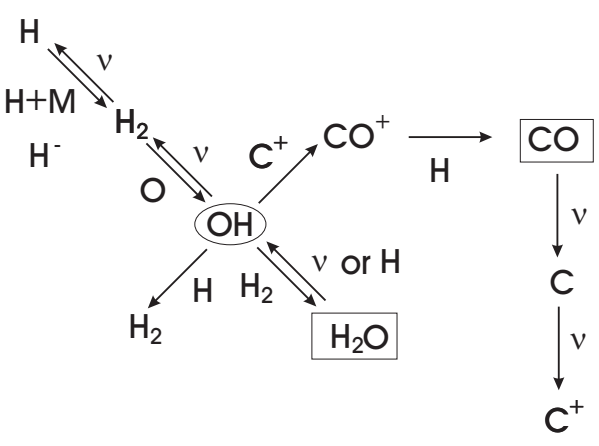

Fig. 6. Schematic representation of the proposed chemical network. $\mathrm{OH}$ radicals play a central role in this network. $v$ means photodissociation by ultraviolet photons and $\mathrm{M}$ a third body $(\mathrm{M}=\mathrm{H}, \mathrm{He})$. The main routes leading to $\mathrm{CO}$ and $\mathrm{H}_{2} \mathrm{O}$ are shown here.

\subsubsection{Formation of $\mathrm{H}_{2}$}

Molecular hydrogen is the starting molecule for this chemical network. In the absence of dust grains, $\mathrm{H}_{2}$ is slowly synthesized in the gas phase via three possible routes depending on the density and temperature. At moderate densities, the reaction

$\mathrm{H}^{-}+\mathrm{H} \rightarrow \mathrm{H}_{2}+\mathrm{e}-$ 

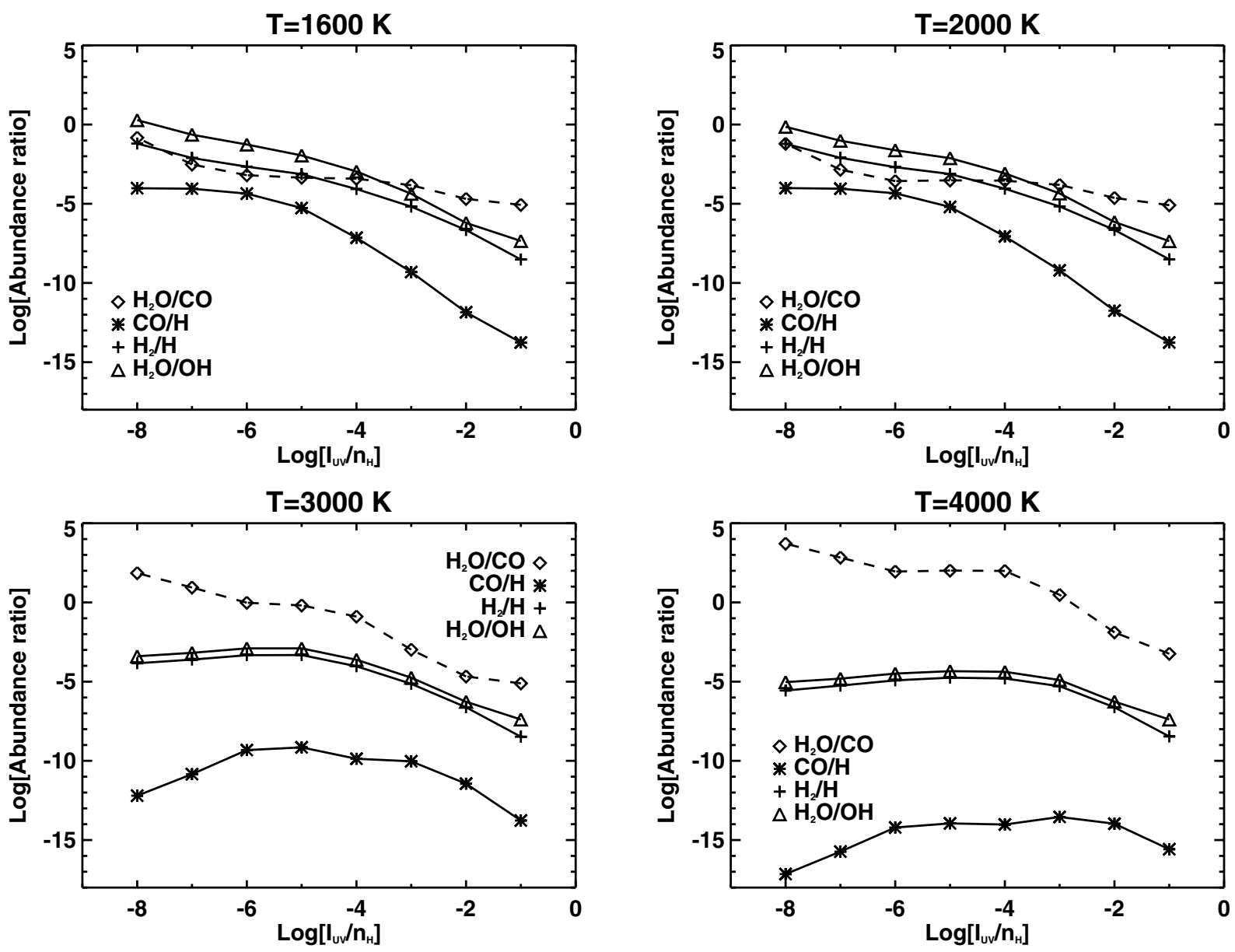

Fig. 7. Relative abundance of various species as a function of $I_{\mathrm{UV}} / n_{\mathrm{H}}$ for gas at temperature $1600 \mathrm{~K}, 2000 \mathrm{~K}, 3000 \mathrm{~K}, 4000 \mathrm{~K}$.

dominates. This reaction has a flat rate of $1.30 \times 10^{-9} \mathrm{~cm}^{3} \mathrm{~s}^{-1}$. At high temperature, $\mathrm{H}_{2}$ formation is initiated by the radiative association:

$\mathrm{H}^{+}+\mathrm{H} \rightarrow \mathrm{H}_{2}^{+}+$photon

which has an activation energy of $8500 \mathrm{~K}$, followed by

$\mathrm{H}_{2}^{+}+\mathrm{H} \rightarrow \mathrm{H}_{2}+\mathrm{H}^{+}$

$\mathrm{H}_{2}$ molecules further react with atomic oxygen or carbon to form $\mathrm{OH}$ or $\mathrm{CH}$. At temperature higher than $3000 \mathrm{~K}, \mathrm{H}_{2}$ is dissociated by collisions with hydrogen atoms. This reaction has an activation energy of $55000 \mathrm{~K}$. At high densities $\left(n_{\mathrm{H}}>\right.$ $\left.10^{12} \mathrm{~cm}^{-3}\right), \mathrm{H}_{2}$ can also be formed by the three-body reaction

$\mathrm{H}+\mathrm{H}+\mathrm{H} \rightarrow \mathrm{H}_{2}+\mathrm{H}$

which is a highly exothermic reaction, but the third hydrogen atom carries away the excess energy. $\mathrm{H}_{2}$ reacts swiftly with atomic oxygen to form $\mathrm{OH}$, which is the key radical for the synthesis of $\mathrm{CO}$ and $\mathrm{H}_{2}$.

\subsubsection{CO formation and destruction}

CO molecules are readily formed for all values of the UV field enhancement, when the density reaches a value of $10^{6} \mathrm{~cm}^{-3}$ and temperatures below $2500 \mathrm{~K}$. The formation of $\mathrm{CO}$ is initiated by the fast reaction at high temperature (activation energy of $E_{\mathrm{a}}=3150 \mathrm{~K}$ )

$\mathrm{H}_{2}+\mathrm{O} \rightarrow \mathrm{OH}+\mathrm{H}$

followed by the reaction:

$\mathrm{C}+\mathrm{OH} \rightarrow \mathrm{CO}+\mathrm{H}$

which has a rate of $1.10 \times 10^{-10}(T / 100)^{1 / 2} \mathrm{~s}^{-1}$. The other channel

$\mathrm{C}+\mathrm{OH} \rightarrow \mathrm{O}+\mathrm{CH}$

has an activation energy of $14800 \mathrm{~K}$.

Above $3000 \mathrm{~K}$, destruction of $\mathrm{CO}$ is enhanced because reactions with high barrier are activated. Especially, $\mathrm{CO}$ is chemically dissociated at gas temperature greater than $3000 \mathrm{~K}$ by the endothermic reaction:

$\mathrm{H}+\mathrm{CO} \rightarrow \mathrm{C}+\mathrm{OH}$.

This reaction is particularly important since hydrogen is mostly in the atomic form.

At lower UV intensities, $\mathrm{CO}$ reacts with the $\mathrm{OH}^{+}$to form $\mathrm{HCO}^{+}$. Here $\mathrm{OH}^{+}$comes from the reaction between $\mathrm{H}_{3}^{+}$ and atomic oxygen. 
At extreme densities, $\mathrm{CO}$ is formed directly from the neutral-neutral addition $\mathrm{C}+\mathrm{O}$, and the excess energy is carried away by a third body, most likely a hydrogen atom. The main destruction reaction beside photodissociation is the hydrogenation of $\mathrm{CO}$ leading to $\mathrm{HCO}$. If the abundance of $\mathrm{H}_{2}$ is not sufficiently high

$\mathrm{CO}+\mathrm{H}+\mathrm{M} \rightarrow \mathrm{HCO}+\mathrm{M}$.

Other possible reactions are

$\mathrm{CO}+\mathrm{H}_{2}+\mathrm{M} \rightarrow \mathrm{HCO}+\mathrm{H}$,

where $\mathrm{M}$ is a third body (likely $\mathrm{H}$ or $\mathrm{He}$ ). Alternatively, with low $\mathrm{H}_{2}$ abundance, $\mathrm{CO}$ preferably reacts with $\mathrm{OH}$ to form $\mathrm{CO}_{2}$ :

$\mathrm{CO}+\mathrm{OH} \rightarrow \mathrm{CO}_{2}+\mathrm{H}$.

Finally, the reaction

$\mathrm{HCO}+\mathrm{H}+\mathrm{M} \rightarrow \mathrm{H}_{2} \mathrm{CO}+\mathrm{M}$

converts $\mathrm{HCO}$ into $\mathrm{H}_{2} \mathrm{CO}$. Therefore, $\mathrm{HCO}$ and $\mathrm{H}_{2} \mathrm{CO}$ have non-negligible abundance.

\subsubsection{Water molecules}

The chemical reactions leading to water molecules in cold and hot regions are different. In quiescent molecular clouds, the gas temperature is below $230 \mathrm{~K}$, and gas-phase chemistry gives typical $\mathrm{H}_{2} \mathrm{O}$ abundances of a few $\times 10^{-7}$ (e.g., Lee et al. 1996; Le Teuff et al. 2000). Water is formed by a sequence of ion-molecules reactions starting with $\mathrm{O}+\mathrm{H}_{3}^{+}$ or $\mathrm{O}^{+}+\mathrm{H}_{2}$ leading to $\mathrm{OH}^{+}$. Then rapid $\mathrm{H}$-abstraction reactions with $\mathrm{H}_{2}$ produce $\mathrm{H}_{3} \mathrm{O}^{+}$which dissociatively recombines (with electrons) to $\mathrm{H}_{2} \mathrm{O}$. Another potential source of gas phase water molecules is the evaporation of icy grains in regions where $T>100 \mathrm{~K}$. The reaction scheme in cold regions has been proven to be too effective in producing $\mathrm{H}_{2} \mathrm{O}$ when compared to observations of high-mass star forming regions by Snell et al. (2000). At high temperature $\left(T_{\text {gas }}>230 \mathrm{~K}\right)$ and densities lower than $10^{15} \mathrm{~cm}^{-3}$, water molecules are predominately formed via the radical-molecule reaction

$\mathrm{H}_{2}+\mathrm{OH} \rightarrow \mathrm{H}_{2} \mathrm{O}+\mathrm{H}$

since $\mathrm{OH}$ is very abundant. The importance of this reaction has already been realized for water formation in shocks (e.g., Elitzur 1979; Bergin et al. 1998). Water molecules are mostly destroyed by photodissociation and by reaction with $\mathrm{C}^{+}$:

$\mathrm{H}_{2} \mathrm{O}+\mathrm{C}^{+} \rightarrow \mathrm{HOC}^{+} / \mathrm{HCO}^{+}+\mathrm{H}$.

The Bradford sequence for the synthesis of water still plays a small role compared to the radical-molecule formation route described above at the densities attained in inner disks. The reaction network $\left(\mathrm{CO}\right.$ and $\left.\mathrm{H}_{2} \mathrm{O}\right)$ discussed here and summarized in Fig. 6 is similar to those found for post- $J$-shock chemistry (Hollenbach \& McKee 1989). It may therefore be difficult to distinguish between a post-shock or a quiescent hightemperature chemistry origin for many molecules. The production of water vapor by sublimation of the icy mantle when the dust temperature exceeds $100 \mathrm{~K}$ is often advocated to explain a large quantity of $\mathrm{H}_{2} \mathrm{O}$ in the gas phase.

\subsection{Endogenous versus exogenous formation of molecules}

Two main scenarii can explain the presence of molecules in the inner disks around young massive stars. The first model proposes that the molecules are formed at large distances from the central object and subsequently brought to its vicinity by accretion. We call this possibility the exogenous scenario. In the exogenous model, molecular species can also originate from evaporation of icy grain mantle. The second model states that molecules can be readily synthesized locally in the inner disks. This is the endogenous model. All models reach steady-state in at most one year and the effects of the initial molecular abundances are minimal.

\subsection{Effect of $\mathrm{CO}$ self-shielding}

At high column densities, $\mathrm{CO}$ molecules can self-shield from photodissociation (e.g., van Dishoeck \& Black 1988). The nominal column of $\mathrm{CO}$ is $10^{15} \mathrm{~cm}^{-2}$ for a turbulent width of $5 \mathrm{~km} \mathrm{~s}^{-1}$ but this value was derived for gas at low temperature and may not be valid for gas above $1500 \mathrm{~K}$, because highly vibrational excited absorption levels were not taken into account in models of $\mathrm{CO}$ self-shielding. The nominal column of $\mathrm{CO}$ for self-shielding varies as $1 / \Delta v$. For a very turbulent gas with $\Delta v=30 \mathrm{~km} \mathrm{~s}^{-1}$, the nominal column of $\mathrm{CO}$ is increased by a factor $\sim 6$. Nevertheless, we ran models with increasing $\mathrm{CO}$ column density $N(\mathrm{CO})$ to account for probable increase in the value of the nominal $\mathrm{CO}$ for self-shielding needed to simulate the condition in inner disks. The other parameters are $T_{\text {gas }}=1600 \mathrm{~K}, n_{\mathrm{H}}=10^{12} \mathrm{~cm}^{-3}$, and $I_{\mathrm{UV}}=10^{8}$. Figure 10 shows that above the threshold $\mathrm{CO}$ column density of $\times 10^{15} \mathrm{~cm}^{-2}$, CO molecules are self-protected against photodissociation, and their abundance can reach the cold quiescent molecular cloud value of $\sim 10^{-4}$. Surprisingly, $\mathrm{OH}$ and $\mathrm{H}_{2} \mathrm{O}$ abundances seem to decrease when $\mathrm{CO}$ abundance rises steeply. The $\mathrm{H}_{2} \mathrm{O} / \mathrm{CO}$ ratio drops dramatically from few $10^{-4}$ to few $10^{-7}$. From Fig. 8, it is clear that the effect of CO selfshielding is most noticeable for $I_{\mathrm{UV}} / n_{\mathrm{H}}>10^{-5}$ (the photodominated regime). The $\mathrm{CO}$ abundance reaches similar value to the thermal region, i.e. where $I_{\mathrm{UV}} / n_{\mathrm{H}}<10^{-5}$.

\subsection{Effect of $\mathrm{C} / \mathrm{O}$ abundance ratio}

By pushing the dust grains beyond the dust sublimation radius, the radiation pressure will separate gas and dust. Segregation of the gas and dust results in an increase in elemental C/O ratio because the silicate grains, which contain $10-20 \%$ of the available oxygen, do not sublimate. The effect of an increase in the elemental $\mathrm{C} / \mathrm{O}$ ratio above the solar value of 0.4 would be to lower the water and molecular oxygen abundance. In theory, the elemental abundances can be deduced from the study of the star's photospheric lines if it is not too embedded. Terzieva \& Herbst (1998) discussed the different effects on the chemistry caused by variations in the $\mathrm{C} / \mathrm{O}$ elemental abundance, but their study is limited to low temperature chemistry where elements can be depleted onto grains. Here we allow the oxygen's elemental abundance to vary from 0.1 

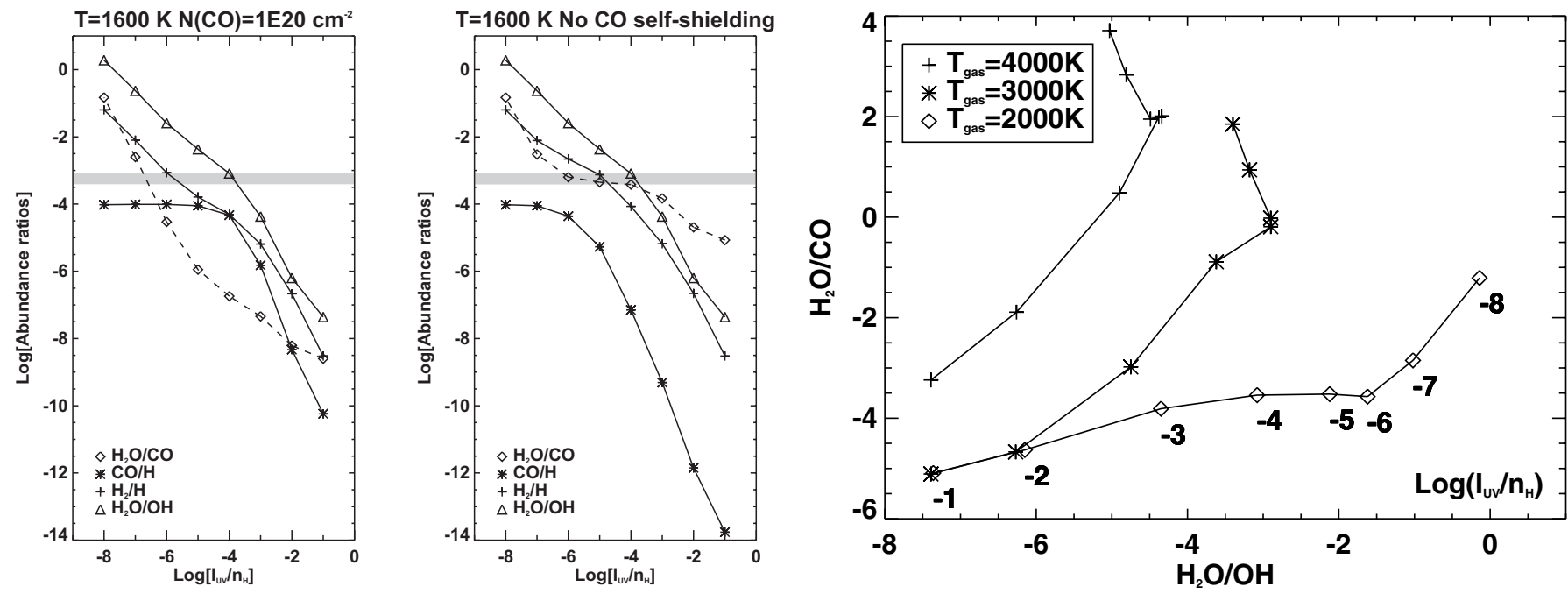

Fig. 8. Effect of CO self-shielding on abundance ratios. The left panel shows the addition of a strong self-shielding on the chemistry, while the right panel is the same as the upper right panel in Fig. 7. As expected the changes happen only on the $\mathrm{H}_{2} \mathrm{O} / \mathrm{CO}$ and $\mathrm{CO} / \mathrm{H}$ ratios. The observed value of $\mathrm{H}_{2} \mathrm{O} / \mathrm{CO}\left(\sim 5.4 \times 10^{-4}\right)$ is indicated by a gray strip.

to 1.0 times the nominal value listed in Table 6 . The model results at steady-state are shown in Fig. 11. As expected, the relative abundance of $\mathrm{CO}, \mathrm{OH}$, and $\mathrm{H}_{2} \mathrm{O}$ diminish with decreasing oxygen elemental abundance. The rate of decrease is similar for the three species. Therefore, the relative abundances $\mathrm{H}_{2} \mathrm{O} / \mathrm{CO}$ and $\mathrm{H}_{2} \mathrm{O} / \mathrm{OH}$ are not significantly affected by changing the oxygen abundance. The simultaneous decrease in $\mathrm{CO}$ and $\mathrm{H}_{2} \mathrm{O}$ abundance with lower oxygen elemental abundance can be ascribed to the formation route of both molecules that are formed via the radial $\mathrm{OH}$. This contrasts with the chemistry that occurs in quiescent molecular clouds where $\mathrm{H}_{2} \mathrm{O}$ is synthesized at a much lower rate than $\mathrm{CO}$, which then results in a higher $\mathrm{CO} / \mathrm{H}_{2} \mathrm{O}$ ratio as the oxygen elemental abundance decreases.

\subsection{Comparison with the observations}

A large range of gas temperature (1600-4800 K) has been inferred from modeling CO bandhead emission toward four high-mass young stars (Bik \& Thi 2004). The abundance of $\mathrm{CO}$ starts to decrease at temperature above $2500 \mathrm{~K}$ owing to collisional dissociation of $\mathrm{CO}$ and to the reaction of $\mathrm{CO}$ with $\mathrm{OH}$ to form $\mathrm{CO}_{2}$.

The observed $\mathrm{H}_{2} \mathrm{O} / \mathrm{CO}$ ratio in $08576 \mathrm{nr} 292$ is $5.4 \times$ $10^{-4}$. If $\mathrm{CO}$ self-shielding is taken into account, the value for $I_{\mathrm{UV}} / n_{\mathrm{H}}$ is not well-determined because the theoretical curve for $\mathrm{H}_{2} \mathrm{O} / \mathrm{CO}$ is relatively flat around few times $10^{-4}$ (upper left panel in Fig. 7). The $\mathrm{CO}$ abundance is $10^{-7}-10^{-4}$ and $\mathrm{H}_{2} \mathrm{O}$ abundance is $10^{-11}-10^{-8}$. Interestingly, when $\mathrm{CO}$ is allowed to self-shield, we can infer $I_{\mathrm{UV}} / n_{\mathrm{H}} \sim 10^{-7}$ for a gas at $T=1600 \mathrm{~K}$ better from the observed value of $\mathrm{H}_{2} \mathrm{O} / \mathrm{CO}$ since the "degeneracy" in the $\mathrm{H}_{2} \mathrm{O} / \mathrm{CO}$ curve in the right panel of Fig. 8 is lifted when $\mathrm{CO}$ self-shielding is taken into account in the modeling.

Fig. 9. $\mathrm{H}_{2} \mathrm{O} / \mathrm{CO}$ versus $\mathrm{H}_{2} \mathrm{O} / \mathrm{OH}$ diagram for various $I_{\mathrm{UV}} / n_{\mathrm{H}}$ and gas temperature.

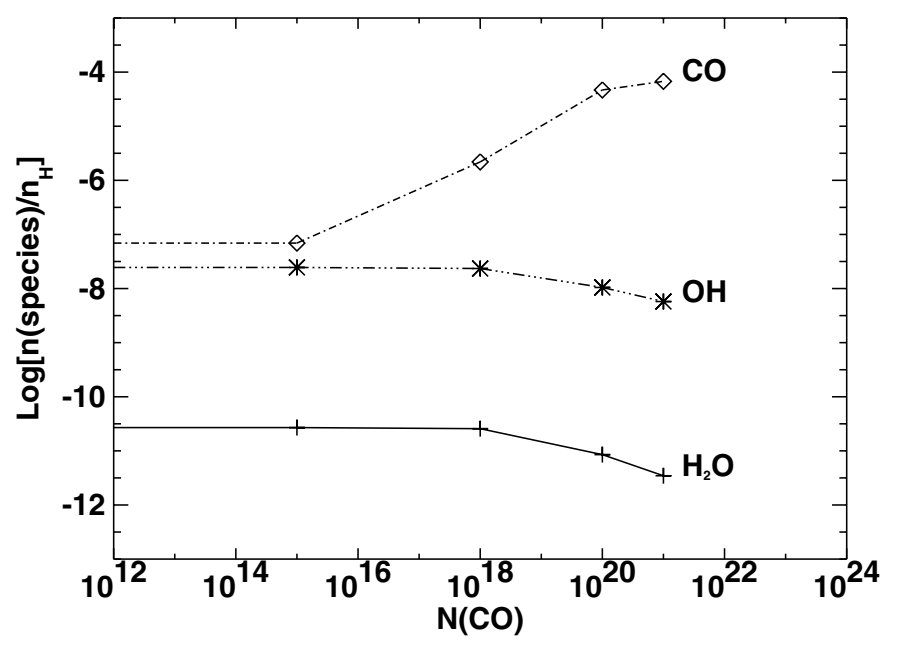

Fig. 10. Effect of the CO self-shielding on the relative abundances. The self-shielding factor depends on the $\mathrm{CO}$ column density $N(\mathrm{CO})$. The parameters of the model are $T_{\text {gas }}=1600 \mathrm{~K}, n_{\mathrm{H}}=10^{12} \mathrm{~cm}^{-3}$, and $I_{\mathrm{UV}}=10^{8}$.

The absolute abundances are low in comparison to that in the envelope of high-mass young stellar objects (Boonman et al. 2003). From the CO abundance in the unshielded case, we infer a total hydrogen column density of $3.9 \times 10^{25}-10^{28} \mathrm{~cm}^{-2}$ or surface density $\Sigma_{0}=6.5-65000 \mathrm{~g} \mathrm{~cm}^{-2}$. When CO selfshielding effects are included in the modeling, $\mathrm{CO} / \mathrm{H}$ reaches the maximum relative abundance of $10^{-4}$ and the total hydrogen column density amounts to $3.9 \times 10^{25} \mathrm{~cm}^{-2}$ or a surface density of $\Sigma_{0}=6.5 \mathrm{~g} \mathrm{~cm}^{-2}$. We provide a large possible range for the derived total hydrogen column density, because current $\mathrm{CO}$ self-shielding models may not be applicable to the situation encountered in inner disks. However, it is noteworthy that the upper range of column density $\left(N(\mathrm{H})>10^{26} \mathrm{~cm}^{-2}\right)$ would imply large opacity in the near-infrared due to $\mathrm{H}^{-}$, which would prevent the detection of optically thin $\mathrm{H}_{2} \mathrm{O}$ lines. The surface density is comparable to the values adopted in 


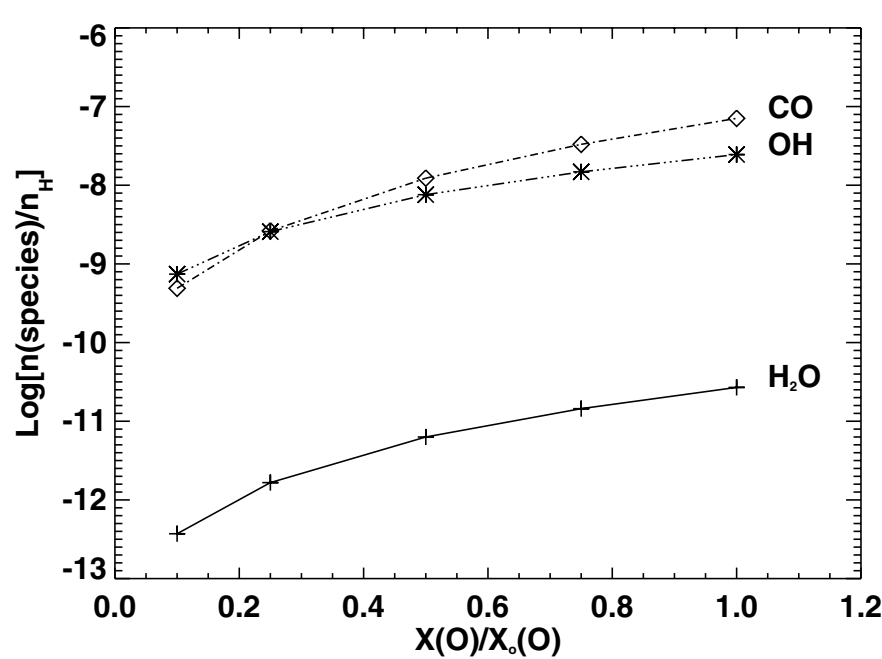

Fig. 11. Effect of the oxygen elemental abundance on the relative abundance of $\mathrm{CO}, \mathrm{OH}$, and $\mathrm{H} 2 \mathrm{O} . X_{\mathrm{o}}(\mathrm{O})$ is the nominal value of the Oxygen elemental abundance. The parameters of the model are $T_{\text {gas }}=1600 \mathrm{~K}, n_{\mathrm{H}}=10^{12} \mathrm{~cm}^{-3}$, and $I_{\mathrm{UV}}=10^{8}$.

disk models around $\mathrm{T}$ Tauri and Herbig Ae stars, which is $\Sigma_{0} \sim 10^{2}-10^{4} \mathrm{~g} \mathrm{~cm}^{-2}$ at $1 \mathrm{AU}$.

We summarize the chemical modeling in Fig. 9. This figure shows the $\mathrm{H}_{2} \mathrm{O} / \mathrm{CO}$ versus $\mathrm{H}_{2} \mathrm{O} / \mathrm{OH}$ abundance ratio. The figure provides a simple way to deduce physical parameters such as $T_{\text {gas }}$ and $I_{\mathrm{UV}} / n_{\mathrm{H}}$ from $\mathrm{CO}, \mathrm{OH}$, and $\mathrm{H}_{2} \mathrm{O}$ abundance. Unfortunately, $\mathrm{OH}$ lines were not covered in the $K$-band spectrum of $08576 \mathrm{nr} 292$.

\subsection{Comparison with the inner disk chemistry around low-mass young stars}

Glassgold et al. (2004) studied the thermal-chemical structure of the inner disks around young low mass stars. Although in low-mass young stars X-rays drive the chemistry rather than the UV, they found that the disk atmosphere may contain a significant amount of warm $(T=500-2000 \mathrm{~K}) \mathrm{CO}$ gas similar to our results. This similarity is not surprising since at the high temperatures and densities encountered in inner disks around low- and high-mass young stars, the chemistry is dominated by the same neutral-neutral reactions. At very high densities, the UV and X-ray photons provide the energy needed to maintain a high temperature. The photodissociation by X-ray or UV photons of the molecules are compensated for by rapid formation in dust-free regions. There, X-ray and UV photons are absorbed by gas, while in a dust-rich region UV radiation is rapidly attenuated by dust absorption while the X-ray can penetrate deeper to influence the ionization fraction (e.g., Semenov et al. 2003).

\section{Conclusion and future prospects}

We have shown that hot-water vapor emission is present in the close vicinity $(R<5 \mathrm{AU})$ of the high-mass young stellar object 08576nr292. The water vapor, as well as hot CO, are most likely located in a disk in Keplerian rotation.

The detection of optically thin $\mathrm{CO}$ and $\mathrm{H}_{2} \mathrm{O}$ lines at $1500-1600 \mathrm{~K}$ suggests that dust grains are absent in that temperature range in the disk, which may stem from the fact that dust grains are pushed further out by radiation pressure or that most dust has evaporated. The region probed by hot $\mathrm{CO}$ and $\mathrm{H}_{2} \mathrm{O}$ is limited to the inner few $\mathrm{AU}$ of the disk. Alternatively, the gas and dust may be thermally decoupled with the gas much warmer than the dust.

To probe cooler gas, which is located between 1 and $\sim 50 \mathrm{AU}$, fundamental transitions of $\mathrm{CO}$ and lower $J$ lines of $\mathrm{H}_{2} \mathrm{O}$ appear to be more appropriate, although the line over continuum contrast would be smaller owing to the warm dust emission. Nevertheless, hot lines allow the gas-rich and probably dust-poor region to be investigated. Higher resolution spectrometers $\left(R=100000\right.$ or $\left.\Delta v=3 \mathrm{~km} \mathrm{~s}^{-1}\right)$, such as CRIRES planned for the $V L T$, will resolve the individual $\mathrm{H}_{2} \mathrm{O}$ line profiles. The combination of medium resolution $(R=10000)$ and high spatial resolution achieved by $A M B E R$ will help to determine the exact location of both the $\mathrm{CO}$ and $\mathrm{H}_{2} \mathrm{O}$ emissions.

Chemical models have been developed for the dense and hot inner disk around young high-mass stars. The chemistry is dominated by neutral-neutral reactions and steady-state is rapidly attained. $\mathrm{H}_{2}$ formation is hampered by the lack of dust grains and reaches relatively low abundance. The radical $\mathrm{OH}$ is central to the chemical network playing the same role as $\mathrm{H}_{3}^{+}$for cold chemistry. The molecular abundances depend in general on the gas temperature and UV flux over density ratio and show a large range of values. Photodissociation affects more $\mathrm{H}_{2}, \mathrm{OH}$, and $\mathrm{H}_{2} \mathrm{O}$ than $\mathrm{CO}$ because the latter species can self-shield very efficiently. The observed $\mathrm{H}_{2} \mathrm{O} / \mathrm{CO}$ can be reproduced at the temperature of the gas $(1600 \mathrm{~K})$ and $I_{\mathrm{UV}} / n_{\mathrm{H}} \sim 10^{-6}$. Observations of $\mathrm{OH}$ are warranted in order to allow better constraints on the UV radiation field. Future work on the modeling side includes the combination of the chemical model presented here with a dynamic disk model around young high-mass stars.

Detection of molecules such as $\mathrm{CO}$ and $\mathrm{H}_{2} \mathrm{O}$ at high temperature in the close vicinity of young massive young stellar objects opens the possibility of studying the physics and chemistry in the region where matter from the disk falls onto the star.

Acknowledgements. W.F.T. was supported by NWO grant 614.041.005 during his stay in Amsterdam. The authors thank the VLT staff for performing the observations in Service mode. Comments and suggestions from Rens Waters and Lex Kaper are much appreciated.

\section{References}

Aikawa, Y., Umebayashi, T., Nakano, T., \& Miyama, S. M. 1999, ApJ, 519,705

Allard, F., Hauschildt, P. H., Alexander, D. R., Tamanai, A., \& Schweitzer, A. 2001, ApJ, 556, 357

Bergin, E. A., Melnick, G. J., Stauffer, J. R., et al. 2000, ApJ, 539, L129

Bergin, E. A., Neufeld, D. A., \& Melnick, G. J. 1998, ApJ, 499, 777

Bernath, P. F. 1995, Spectra of Atoms and Molecules (NY: Oxford University Press), 400

Bevington, P. R., \& Robinson, D. K. 2003, Data reduction and error analysis for the physical sciences, 3rd ed., P. R. Bevington, and K. D. Robinson (Boston, MA: McGraw-Hill), ISBN 0-07-247227-8 
Bik, A., Kaper, L., Waters, L. B. F. M., \& Hanson, M. M. 2004, A\&A, submitted

Bik, A., \& Thi, W. F. 2004, A\&A, 427, L13

Blake, G. A., \& Boogert, A. C. A. 2004, ApJ, 606, L73

Blum, R. D., Barbosa, C. L., Damineli, A., \& Conti, P. I. 2005, ApJ [arXiv: astro-ph/0409190]

Bonnell, I. A., \& Bate, M. R. 2002, MNRAS, 336, 659

Boonman, A. M. S., Doty, S. D., van Dishoeck, E. F., et al. 2003, A\&A, 406, 937

Brittain, S. D., Rettig, T. W., Simon, T., et al. 2003, ApJ, 588, 535

Brown, P. N., Byrne, G. D., \& Hindmarsh, A. C. 1989, SIAM J. Sci. Stat. Comput., 10, 1038

Burton, M. G., Hollenbach, D. J., \& Tielens, A. G. G. M. 1990, ApJ, 365,620

Carr, J. S., Tokunaga, A. T., \& Najita, J. 2004, ApJ, 603, 213

Chandler, C. J., Carlstrom, J. E., \& Scoville, N. Z. 1995, ApJ, 446, 793

Charbonneau, P. 1995, ApJS, 101, 309

Churchwell, E. 2002, ARA\&A, 40, 27

Elitzur, M. 1979, ApJ, 229, 560

Glassgold, A. E., Najita, J., \& Igea, J. 2004, ApJ, 615, 972

Helmich, F. P., van Dishoeck, E. F., Black, J. H., et al. 1996, A\&A, 315, L173

Hollenbach, D., \& McKee, C. F. 1989, ApJ, 342, 306

Jijina, J., \& Adams, F. C. 1996, ApJ, 462, 874

Kamp, I., van Zadelhoff, G.-J., van Dishoeck, E. F., \& Stark, R. 2003, A\&A, 397, 1129

Kaper, L., Bik, A., Comerón, F., \& Hanson, M. M. 2005, A\&A, submitted

Kaufman, M. J., \& Neufeld, D. A. 1996, ApJ, 456, 611

Kraus, M., Krügel, E., Thum, C., \& Geballe, T. R. 2000, A\&A, 362, 158

Lamy, P. L. 1974, A\&A, 35, 197

Le Teuff, Y. H., Millar, T. J., \& Markwick, A. J. 2000, A\&AS, 146, 157

Lee, H.-H., Bettens, R. P. A., \& Herbst, E. 1996, A\&AS, 119, 111

Lenorzer, A., Bik, A., de Koter, A., et al. 2004, A\&A, 414, 245

Lewis, J. S., \& Prinn, R. G. 1980, ApJ, 238, 357

Liseau, R., Lorenzetti, D., Nisini, B., Spinoglio, L., \& Moneti, A. 1992, A\&A, 265, 577
Markwick, A. J., Ilgner, M., Millar, T. J., \& Henning, T. 2002, A\&A, 385,632

Melnick, G. J., Ashby, M. L. N., Plume, R., et al. 2000, ApJ, 539, L87

Mundy, L. G., Looney, L. W., \& Welch, W. J. 2000, Protostars and Planets IV, 355

Najita, J., Carr, J. S., Glassgold, A. E., Shu, F. H., \& Tokunaga, A. T. 1996, ApJ, 462, 919

Najita, J., Carr, J. S., \& Mathieu, R. D. 2003, ApJ, 589, 931

Natta, A., Grinin, V., \& Mannings, V. 2000, Protostars and Planets IV, 559

Nguyen, T. K., Viti, S., \& Williams, D. A. 2002, A\&A, 387, 1083

Press, W. H., Teukolsky, S. A., Vetterling, W. T., \& Flannery, B. P. 1992, Numerical recipes in FORTRAN. The art of scientific computing (Cambridge: University Press), 2nd ed.

Rothman, L. S., Barbe, A., Benner, D. C., et al. 2003, J. Quant. Spectrosc. Rad. Transfer, 82, 5

Saija, R., Iatì, M. A., Giusto, A., et al. 2003, MNRAS, 341, 1239

Scoville, N., Kleinmann, S. G., Hall, D. N. B., \& Ridgway, S. T. 1983, ApJ, 275, 201

Semenov, D., Henning, T., Helling, C., Ilgner, M., \& Sedlmayr, E. 2003, A\&A, 410, 611

Semenov, D., Wiebe, D., \& Henning, T. 2004, A\&A, 417, 93

Snell, R. L., Howe, J. E., Ashby, M. L. N., et al. 2000, ApJ, 539, L97

Tennyson, J., Zobov, N. F., Williamson, R., Polyansky, O. L., \& Bernath, P. F. 2001, J. Phys. Chem. Ref. Data, 30, 735

Terzieva, R., \& Herbst, E. 1998, ApJ, 501, 207

van Dishoeck, E. F., \& Black, J. H. 1988, ApJ, 334, 771

Walsh, A. J., Burton, M. G., Hyland, A. R., \& Robinson, G. 1999, MNRAS, 309, 905

Waters, L. B. F. M., \& Waelkens, C. 1998, ARA\&A, 36, 233

Weingartner, J. C., \& Draine, B. T. 2001, ApJ, 548, 296

Wolfire, M. G., \& Cassinelli, J. P. 1987, ApJ, 319, 850

Wright, C. M., van Dishoeck, E. F., Black, J. H., et al. 2000, A\&A, 358,689

Yorke, H. W., \& Sonnhalter, C. 2002, ApJ, 569, 846

Zobov, N. F., Polyansky, O. L., Tennyson, J., et al. 2000, ApJ, 530, 994 